\title{
A Novel SHLNN Based Robust Control and Tracking Method for Hypersonic Vehicle under Parameter Uncertainty
}

\author{
Chuanfeng Li, ${ }^{1,2}$ Hao Wu, ${ }^{3}$ Zhile Yang, ${ }^{4}$ Yongji Wang, ${ }^{5}$ and Zeyu Sun ${ }^{1}$ \\ ${ }^{1}$ School of Computer and Information Engineering, Luoyang Institute of Science and Technology, Luoyang 471023, China \\ ${ }^{2}$ School of Electronics, Electrical Engineering and Computer Science, Queen's University Belfast, Belfast BT9 5AH, UK \\ ${ }^{3}$ Beijing Aerospace Automation Control Institute, Beijing 100854, China \\ ${ }^{4}$ Shenzhen Institute of Advanced Technology, Chinese Academy of Sciences, Shenzhen, Guangdong 518055, China \\ ${ }^{5}$ School of Automation, Huazhong University of Science and Technology, Wuhan 430074, China
}

Correspondence should be addressed to Zhile Yang; zyang07@qub.ac.uk

Received 6 July 2017; Accepted 11 September 2017; Published 17 October 2017

Academic Editor: Guang Li

Copyright (c) 2017 Chuanfeng Li et al. This is an open access article distributed under the Creative Commons Attribution License, which permits unrestricted use, distribution, and reproduction in any medium, provided the original work is properly cited.

Hypersonic vehicle is a typical parameter uncertain system with significant characteristics of strong coupling, nonlinearity, and external disturbance. In this paper, a combined system modeling approach is proposed to approximate the actual vehicle system. The state feedback control strategy is adopted based on the robust guaranteed cost control (RGCC) theory, where the Lyapunov function is applied to get control law for nonlinear system and the problem is transformed into a feasible solution by linear matrix inequalities (LMI) method. In addition, a nonfragile guaranteed cost controller solved by LMI optimization approach is employed to the linear error system, where a single hidden layer neural network (SHLNN) is employed as an additive gain compensator to reduce excessive performance caused by perturbations and uncertainties. Simulation results show the stability and well tracking performance for the proposed strategy in controlling the vehicle system.

\section{Introduction}

The long-distance unpowered glide reentry vehicle is an important hypersonic vehicle which has been of significant aerodynamic configuration with high lift-to-drag ratio. It can reach the target after long-distance gliding and fulfill a throwing mission through reentering from orbit or suborbit. Given strong ability to fulfill high-speed remote precision attack and power projection, this vehicle is of great implication function for strategic planning. However, the vehicle is a complex nonlinear object, and how to design the control strategy to ensure the stability of vehicle system has become a crucial topic [1-6].

Conventional technologies are majorly based on performing time-domain simulation and relied heavily on the results of human experience. Since the birth of modern control theory in the 1950s, control theory develops rapidly and has been successfully adopted in the aerospace application in the 1960s $[7,8]$. In the recent few decades, robust control has gained remarkable attentions due to the well adaptation ability in dealing with objects in uncertain and noisy environment [9-13]. With the maturation of robust control theory, Kharitonov interval theory, Ho control theory, and structural singular value theory ( $\mu$ theory) have been widely used in aircraft controller design and trajectory tracking. For instance, the refinement of the existing method by considering 16 segment plants instead of 16 Kharitonov plants provides an efficient tool for designing all robustly stabilizing PID controllers for an interval system [14]. An $H \infty$ method for designing reduced-order output-feedback controllers for linear time-invariant retarded systems was introduced to achieve a minimum bound on the $H$-infinity performance level [15]. The clearance of flight control law for a hypersonic gliding vehicle (HGV) and two linear clearance criteria based on structural singular value $(\mu)$ theory were proposed in [16]. However, since the aircraft is a nonlinear system, of which the mathematical model has parametric uncertainties, it is straightforward to deviate from the actual control by using direct linearization method. 
Assume that the system is in instantaneous equilibrium, basic formula of linearized equations is then applied, and small deviation model of simplified equations is achieved [17]. In order to consider the effects of nonlinearity on the system, the article [18] shows the identifiability of a nonlinear delayed-differential model describing aircraft dynamics. In order to reduce the impact of model parameters perturbation on the system, a mixed $H 2 / H \infty$ control was proposed using fuzzy singularly perturbed model with multiple perturbation parameters [19]. A new strategy for missile attitude control using a hybridization of Linear Quadratic Gaussian (LQG), Loop Transfer Recovery (LTR), and Linear Quadratic Integral (LQI) control techniques was established [20]. However, it will result in relatively conservative results and will undermine the performance robustness of the system, due to the robust LQG control to maintain the minimum performance index. Guaranteed cost control (GCC) on uncertainty system is an effective method to solve the flaws of LQG design [21].

The GCC method can maintain the stability of the closedloop system particularly when the controlled object has significant uncertainty. Meanwhile, it also ensures that the secondary performance index does not exceed the upper bound. A typical application of GCC method for a flexible air-breathing hypersonic vehicle (FAHV) can be found in [22]. In [23], the tracking GCC law was presented combined with the decoupling control to accommodate the parameter uncertainties without coupling. A modified GCC strategy has also been established for discrete-time uncertain systems with both state and input delays [24]. A robust guaranteed cost controller was proposed for quadrotor UAV system with uncertainties to address set-point tracking problem [25]. In order to eliminate disturbance effects and guarantee the robust stability of a quadrotor helicopter with state delay, improved guaranteed cost control and quantum adaptive control were developed [26]. A neural network (NN) based approximate optimal GCC design was developed to find a robust state feedback controller such that the closed-loop system has not only a bounded response in a finite duration of time for all admissible uncertainties but also a minimal guaranteed cost [27].

However, in order to obtain stronger robustness, robust control gains might be sensitive or fragile with respect to some errors or variations in control gains of feedback control. Therefore, a concept of nonfragile control strategy has been proposed, which gives a state feedback controller with enough regulating margin when control gains are variated. In [28], a synchronization problem for complex dynamical networks with additive time-varying coupling delays via nonfragile control was investigated. It has also been concerned with a problem of nonfragile robust optimal guaranteed cost control for a class of uncertain two-dimensional discrete state-delayed systems and the state feedback controllers are designed [29]. Robust nonfragile control of uncertain linear system and application to vehicle active suspension were described in [30]. In [31, 32], nonfragile guaranteed cost control of parametric uncertain systems was studied and the guaranteed cost nonfragile tracking control on the omnidirectional rehabilitative training walker was examined.
Though numerous research methods have been proposed in robust controller design, very limited work has been focused on the application of the hypersonic vehicles. Aiming at the complex hypersonic vehicle nonlinear system, small deviation linear equations are widely used in numerical analysis, but it may lead to the reduced model which can hardly achieve sufficient effect in the application of nonlinear system. The controller remains to be adjusted with considerable efforts before it can guarantee required control index. In this paper, we for the first time propose a linear and nonlinear combination in the course of system modeling, in order to make the expected model closer to the actual system. The Lyapunov function can be applied to get control law for nonlinear system when it satisfies certain Lipschitz conditions, and the problem is transformed into a feasible solution with linear matrix inequalities (LMI) method. Besides, adaptive SHLNN based nonfragile guaranteed cost control strategy is utilized to design the robust controller, with equivalent solution derived from LMI optimization approach. SHLNN are exploited as additive gain adjustments to eliminate the influence of results show that conservative control gains and counteract excessive upper bound of cost function are caused by uncertainties.

The rest of this article is organized as follows. In Section 2, motion model of hypersonic vehicle is formulated, where the state equations of vehicle body are established to testify the effectiveness of the proposed RGCC method. To prove the following theorems, several lemmas and assumptions are described in Section 3. Section 4 demonstrates the robust GCC law in the form of theorem under the Lipschitz conditions. In Section 5, a new adaptive nonfragile robust control strategy is presented, in which a nonfragile guaranteed cost controller solved by LMI optimization approach is applied. In Section 6, SHLNN controller design for nonfragile GCC strategy is treated as an additive gain compensator to reduce excessive performance caused by perturbations and uncertainties. Finally, simulation results of robust control and attitude tracking control are conducted and better stability and tracking performance by the proposed strategies for hypersonic vehicle model are gained.

\section{Kinematics Model of Hypersonic Vehicle}

According to the instantaneous equilibrium condition, the small deviation model of vehicle can be obtained based on the basic formulation of linearization equations. By analyzing motion mechanism and flight characteristics of the hypersonic vehicle, the motion equations of the vehicle body coordinate system are achieved as follows:

$$
\begin{aligned}
& \dot{\alpha}=a_{11} F_{x 1}+a_{12} F_{y 1}+a_{13} \omega_{x 1}+a_{14} \omega_{y 1}+a_{15} \omega_{z 1}, \\
& \dot{\beta}=b_{11} F_{x 1}+b_{12} F_{y 1}+b_{13} F_{z 1}+b_{14} \omega_{x 1}+b_{15} \omega_{y 1}, \\
& \dot{\gamma}=c_{11} \omega_{x 1}+c_{12} \omega_{y 1}+c_{13} \omega_{z 1}, \\
& J_{x 1} \frac{d \omega_{x 1}}{d t}+\left(J_{z 1}-J_{y 1}\right) \omega_{z 1} \omega_{y 1}=M_{x 1},
\end{aligned}
$$




$$
\begin{aligned}
& J_{y 1} \frac{d \omega_{y 1}}{d t}+\left(J_{x 1}-J_{z 1}\right) \omega_{x 1} \omega_{z 1}=M_{y 1} \\
& J_{z 1} \frac{d \omega_{z 1}}{d t}+\left(J_{y 1}-J_{x 1}\right) \omega_{y 1} \omega_{x 1}=M_{z 1}
\end{aligned}
$$

where $\alpha$ represents attack angle, $\beta$ denotes sideslip angle, and $\gamma$ is roll angle. $F_{x 1}, F_{y 1}, F_{z 1}$ represent the components acting on missile body coordinates; $\omega_{x 1}, \omega_{y 1}, \omega_{z 1}$ represent the $\omega$ on $x-, y$-, $z$-axis of missile body coordinates. $J_{x 1}, J_{y 1}$, $J_{z 1}$ are vehicle's moment of inertias relative to each axis of vehicle body coordinate system; $d \omega_{x 1} / d t, d \omega_{y 1} / d t, d \omega_{z 1} / d t$ are components of vehicle rotation angular acceleration vector on each axis, respectively.

In these equations, values of parameters $a, b$, and $c$ are varied in aerodynamic model of vehicle. During the entire flight course, dramatic environmental changes will cause tens or even hundreds of times change of the aerodynamic parameters, which results in significant uncertainties on the mathematical vehicle model.

\section{State Equation Description Form of Hypersonic Vehicle}

Aiming at a classical nonlinear uncertain system, the state equation can be described as follows:

$$
\begin{aligned}
& \dot{x}(t)=\left(A_{1}+\Delta A\right) x(t)+\left(B_{1}+\Delta B\right) u(t)+f(x, t), \\
& y(t)=C x(t) .
\end{aligned}
$$

$x(t) \in R^{n}$ represents state vector, and $x(0)=x_{0} . u(t) \in R^{m}$ is control input vector, and $f(x, t) \in R^{n}$ is a nonlinear part and is a state-related nonlinear function which meets the global Lipschitz condition in Assumption 5. $A_{1}$ and $B_{1}$ are matrices with the certain dimension. $\Delta A$ and $\Delta B$ represent parameter uncertainties, assuming that the uncertainties are norm-bounded, which can be expressed as follows:

$$
\left[\begin{array}{ll}
\Delta A & \Delta B
\end{array}\right]=D F(t)\left[\begin{array}{ll}
E_{1} & E_{2}
\end{array}\right],
$$

where $D \in R^{n \times r}, E_{1} \in R^{q \times n}, E_{2} \in R^{q \times m}$ are known real matrices with specific dimension, which characterize the structure of uncertainty in the system, and $F(t) \in R^{r \times q}$ is an unknown time-varying matrix, but norm-bounded as follows.

$$
\Omega=\left\{F(t) \mid F^{T}(t) F(t) \leq I, \forall t\right\} .
$$

The performance indicator is defined as follows.

$$
J=\int_{0}^{\infty}\left[x^{T}(t) Q x(t)+u^{T}(t) R u(t)\right] d t,
$$

where $Q$ and $R$ are symmetric positive definite weighted matrices.

Lemma 1 (see [33]). For a given symmetric matrix, $F \in R^{n \times n}$ is expressed as

$$
F=\left[\begin{array}{ll}
F_{11} & F_{12} \\
F_{21} & F_{22}
\end{array}\right]
$$

where $F_{11} \in R^{r \times r}, F_{12} \in R^{r \times(n-r)}, F_{21} \in R^{(n-r) \times r}, F_{22} \in$ $R^{(n-r) \times(n-r)}$, and the conclusions are as follows.

(1) $F<0$;

(2) $F_{11}<0, F_{22}-F_{12}^{T} F_{11}^{-1} F_{12}<0$;

(3) $F_{22}<0, F_{11}-F_{12} F_{22}^{-1} F_{12}^{T}<0$.

Lemma 2. For $\sigma_{1}(y)=y^{T} Q_{1} y \geq 0$, assuming there is $\tilde{y} \in R^{m}$, where $\sigma(\tilde{y})>0$, then the equivalent forms are as follows.

(1) $y \in R^{m}$ makes $\sigma_{1}(y) \geq 0, y^{T} Q_{0} y>0$.

(2) $\tau \geq 0$ makes $Q_{0}-\tau Q_{1}>0$.

Corollary 3. When $P>0$ and all $\xi \neq 0, \pi$ satisfying $\pi^{T} \pi \leq$ $\xi^{T} C^{T} C \xi$ is established.

$$
\left[\begin{array}{l}
\xi \\
\pi
\end{array}\right]^{T}\left[\begin{array}{cc}
A^{T} P+P A & P B \\
B^{T} P & 0
\end{array}\right]\left[\begin{array}{l}
\xi \\
\pi
\end{array}\right]<0 .
$$

When $\tau \geq 0$ and $P>0$, then

$$
\left[\begin{array}{cc}
A^{T} P+P A+\tau C^{T} C & P B \\
B^{T} P & -\tau I
\end{array}\right]<0 .
$$

Lemma 4. When $D, E$, and $F(t)$ satisfy the certain dimension real matrices, and $F^{T}(t) F(t) \leq I$, one can get the inequality for $\varepsilon>0$.

$$
D F(t) E+E^{T} F^{T}(t) D^{T} \leq \varepsilon D D^{T}+\varepsilon^{-1} E^{T} E .
$$

Assumption 5. Nonlinear function $f(x, t)$ meets global Lipschitz condition, namely,

$$
\begin{aligned}
\|f(x, t)\| & \leq\|G x(t)\|, \\
\|f(x, t)-f(y, t)\| & \leq\|G(x(t)-y(t))\| .
\end{aligned}
$$

\section{Robust Guaranteed Cost Control of Hypersonic Vehicle}

Theorem 6. According to the parameter uncertain system (2), if $f(x, t)=0$ and it meets the performance (5), then there exists $u(t)=K x(t)$ which satisfies the sufficient and necessary conditions for robust guaranteed cost with parameter uncertain closed-loop system: (1) there exists an appropriate constant $\varepsilon>$ 0 , which makes inequality (11) have a positive definite solution $P>0$; (2) the robustness performance index of closed-loop system meets $J \leq \operatorname{tr}(P)$ at the same time.

$$
\begin{aligned}
\left(A_{1}+\right. & \left.B_{1} K+\Delta A+\Delta B K\right)^{T} P \\
& +P\left(A_{1}+B_{1} K+\Delta A+\Delta B K\right)+Q+K^{T} R K<0 .
\end{aligned}
$$

Corollary 7. Given that formula (11) is satisfied, there exist $P$ and $X$ which establish the existence of appropriate positive 
constant, and matrices $W$ and $X$ satisfy allowable uncertainties [34].

$$
\left[\begin{array}{ccccc}
\Pi & \varepsilon D & \left(E_{1} X+E_{2} W\right)^{T} & X & W^{T} \\
* & -\varepsilon I & 0 & 0 & 0 \\
* & * & -\varepsilon I & 0 & 0 \\
* & * & * & -Q^{-1} & 0 \\
* & * & * & * & -R^{-1}
\end{array}\right]<0
$$

where $\Pi=\left(A_{1} X+B_{1} W\right)^{T}+\left(A_{1} X+B_{1} W\right)$. “*” denotes the transpose of symmetric part in equalities, and the definitions in the following matrix are the same. Furthermore, if inequality (12) has a solution $(W, X)$, it can be described as follows:

$$
u^{*}(t)=W X^{-1} x(t)
$$

This denotes a RGCC law of vehicle system. The performance indicator upper bound is

$$
\bar{J} \leq \operatorname{Trace}\left(X^{-1}\right)=\bar{J}^{*} .
$$

Proof. For system (2), order $f(x, t)=0$, and based on Lemma 4 and (3), inequality (11) can be transformed into

$$
\begin{aligned}
\left(A_{1}\right. & \left.+B_{1} K\right)^{T} P+P\left(A_{1}+B_{1} K\right)+\varepsilon P D D^{T} P \\
& +\varepsilon^{-1}\left(E_{1}+E_{2} K\right)^{T}\left(E_{1}+E_{2} K\right)+Q+K^{T} R K \\
& <0 .
\end{aligned}
$$

For inequality (15), based on Lemma 1, the following linear matrix inequalities can be obtained.

$$
\left[\begin{array}{cccc}
\Pi_{1} & \left(E_{1}+E_{2} K\right)^{T} & I & K^{T} \\
* & -\varepsilon I & 0 & 0 \\
* & * & -Q^{-1} & 0 \\
* & * & * & -R^{-1}
\end{array}\right]<0 .
$$

For inequality (16), multiply it by $\operatorname{diag}\left\{P^{-1}, I, I, I\right\}$, and let $P^{-1}=X, K X=W$; then inequality (12) is obtained based on Lemma 1. We introduce the equation of $V(x(t))=$ $x^{T}(t) P x(t)$; then formula (17) for uncertain closed-loop system is obtained:

$$
\begin{aligned}
\dot{V} & (x(t))=\dot{x}^{T}(t) P x(t)+x^{T}(t) P \dot{x}(t)=x^{T}\left(A_{1}\right. \\
& \left.+B_{1} K+\Delta A+\Delta B K\right)^{T} P x(t)+x^{T} P\left(A_{1}+B_{1} K\right. \\
& +\Delta A+\Delta B K) x(t) \\
& =x^{T}\left[\left(A_{1}+B_{1} K+\Delta A+\Delta B K\right)^{T} P\right. \\
& \left.+P\left(A_{1}+B_{1} K+\Delta A+\Delta B K\right)\right] x(t) .
\end{aligned}
$$

We know from Theorem 6 that

$$
\begin{aligned}
\dot{V}(x(t)) & <-x^{T}(t)\left(Q+K^{T} R K\right) x(t) \\
\int_{0}^{\infty} \dot{V}(x(t)) d t & =V(x(\infty))-V(x(0)) \\
& <-\int_{0}^{\infty} x^{T}(t)\left(Q+K^{T} R K\right) x(t) d t
\end{aligned}
$$

On the basis of the stability condition of system, we get $V(x(\infty))=0$; then

$$
\begin{aligned}
& \int_{0}^{\infty} x^{T}(t)\left(Q+K^{T} R K\right) x(t) d t<V(x(0)) \\
& =x^{T}(0) P x(0) .
\end{aligned}
$$

Thereupon we get in a further way

$$
\bar{J} \leq E\{V(x(0))\} \operatorname{Trace}\left(X^{-1}\right)=\bar{J}^{*} .
$$

Proof is over.

Theorem 8. Aiming at uncertain nonlinear system (2) as well as index (5), if there are matrices $X, Y$ and quantity $\varepsilon>0$, $\tau>0$, the following inequality will hold:

$$
\left[\begin{array}{ccccccc}
\Theta_{1} & \tau I & \varepsilon D & \Theta_{2} & X Q & W^{T} R & X G^{T} \\
* & -\tau I & 0 & 0 & 0 & 0 & 0 \\
* & * & -\varepsilon I & 0 & 0 & 0 & 0 \\
* & * & * & -\varepsilon I & 0 & 0 & 0 \\
* & * & * & * & -Q & 0 & 0 \\
* & * & * & * & * & -R & 0 \\
* & * & * & * & * & * & -\tau I
\end{array}\right]<0,
$$

where $\Theta_{1}=A_{1} X+X A_{1}^{T}+B_{1} W+W^{T} B_{1}^{T}, \Theta_{2}=\left(E_{1} X+E_{2} W\right)^{T}$.

Then $u=K x(t)$ is RGCC control law of system (2), where $K=W X^{-1}$, and performance index is $J^{*} \leq x^{T}(0) X^{-1} x(0)$.

Proof. Considering function $V(x(t))=x^{T}(t) P x(t)$, we take $u=K x(t)$ into $(2)$ :

$$
\begin{aligned}
\dot{V} & (x(t))+x^{T}(t) Q x(t)+x^{T}(t) K^{T} R K x(t)=\dot{x}^{T}(t) \\
\cdot & P x(t)+x^{T}(t) P \dot{x}(t)+x^{T}(t) Q x(t)+x^{T}(t) \\
\cdot & K^{T} R K x(t)=\left[x(t)\left(A_{1}+B_{1} K+\Delta A+\Delta B K\right)\right. \\
+ & \left.f^{T}(x, t)\right] P x(t)+x^{T}(t) \\
\cdot & P\left[\left(A_{1}+B_{1} K+\Delta A+\Delta B K\right) x(t)+f(x, t)\right] \\
& +x^{T}(t) Q x(t)+x^{T}(t) K^{T} R K x(t)=x^{T}(t) \\
& \cdot\left[\left(A_{1}+B_{1} K+\Delta A+\Delta B K\right)^{T} P\right.
\end{aligned}
$$




$$
\begin{aligned}
& \left.+P\left(A_{1}+B_{1} K+\Delta A+\Delta B K\right)\right] x(t)+x^{T}(t) \\
& \cdot P f(x, t)+f^{T}(x, t) P x(t)+x^{T}(t) Q x(t) \\
& +x^{T}(t) K^{T} R K x(t) .
\end{aligned}
$$

$\operatorname{Order} \zeta^{T}(t)=\left[\begin{array}{ll}x^{T}(t) & f^{T}(x, t)\end{array}\right]$; then

$$
\begin{aligned}
\dot{V} & (x(t))+x^{T}(t) Q x(t)+x^{T}(t) K^{T} R K x(t) \\
& =z^{T}(t)\left(\begin{array}{ll}
\Omega & P \\
P & 0
\end{array}\right) z(t),
\end{aligned}
$$

where $\Omega=\left(A_{1}+B_{1} K+\Delta A+\Delta B K\right)^{T} P+P\left(A_{1}+B_{1} K+\Delta A+\right.$ $\Delta B K)+Q+K^{T} R K$.

Based on Assumption 5 we get

$$
\zeta^{T}(t)\left[\begin{array}{cc}
-G^{T} G & 0 \\
0 & I
\end{array}\right] \zeta(t) \leq 0 .
$$

Considering Lemma 2 and Corollary 3 , when $\tau_{0}>0$, we can get

$$
\left[\begin{array}{ll}
\Omega & P \\
P & 0
\end{array}\right]-\tau_{0}\left[\begin{array}{cr}
-G^{T} G & 0 \\
0 & I
\end{array}\right]<0 .
$$

Then,

$$
\dot{V}(x(t))+x^{T}(t) Q x(t)+x^{T}(t) K^{T} R K x(t)<0 .
$$

Namely,

$$
\left[\begin{array}{cc}
\Omega+\tau_{0} G^{T} G & P \\
P & -\tau_{0} I
\end{array}\right]<0 .
$$

Multiplying $\operatorname{diag}\left(P^{-1} I\right)$ on inequality (27) left and right, we have

$$
\left[\begin{array}{cc}
P^{-1}\left(\Omega+\tau_{0} G^{T} G\right) P^{-1} & I \\
I & -\tau_{0} I
\end{array}\right]<0 .
$$

Multiplying inequality (28) by $\operatorname{diag}\left(I \tau_{0}^{-1} I\right)$ from both sides, we get

$$
\left[\begin{array}{cc}
P^{-1}\left(\Omega+\tau_{0} G^{T} G\right) P^{-1} & \tau_{0}^{-1} I \\
\tau_{0}^{-1} I & -\tau_{0}^{-1} I
\end{array}\right]<0 .
$$

Order $P^{-1}=X, K X=W$, and $\tau_{0}^{-1}=\tau$, and based on Lemma 4 , we get

$$
\left[\begin{array}{cc}
\Xi & \tau I \\
\tau I & -\tau I
\end{array}\right]<0
$$

where

$$
\begin{aligned}
\Xi= & A_{1} X+X A_{1}^{T}+B_{1} W+W^{T} B_{1}^{T}+\varepsilon_{1} D D^{T}+\varepsilon_{2} D D^{T} \\
& +\varepsilon_{1}^{-1}\left(E_{1} X\right)^{T}\left(E_{1} X\right)+\varepsilon_{2}^{-1}\left(E_{2} W\right)^{T}\left(E_{2} W\right) \\
& +X Q X+W^{T} R W+\tau^{-1} X G^{T} G X .
\end{aligned}
$$

Based on Lemma 1 we know that (30) and (21) are equivalent. The proof is finished. Then from (26), we get

$$
\dot{V}(x(t))<-x^{T}(t)\left(Q+K^{T} R K\right) x(t)<0 .
$$

Under this condition, the system is stable. Integrating both sides of formula (32), we have

$$
\begin{aligned}
\int_{0}^{\infty} \dot{V}(x(t)) d t & =V(x(\infty))-V(x(0)) \\
& <-\int_{0}^{\infty} x^{T}(t)\left(Q+K^{T} R K\right) x(t) d t .
\end{aligned}
$$

According to system stability conditions, $V(x(\infty))=0$; then

$$
\begin{aligned}
& \int_{0}^{\infty} x^{T}(t)\left(Q+K^{T} R K\right) x(t) d t<V(x(0)) \\
& \quad=x^{T}(0) P x(0) .
\end{aligned}
$$

That is,

$$
J^{*} \leq E\{V(x(0))\}=E\left\{x^{T}(0) P x(0)\right\}=\operatorname{tr}\left(X^{-1}\right) .
$$

Theorem 9. Towards uncertain system (2) as well as performance index (5), the following optimization problem

$$
\begin{aligned}
& \min _{\varepsilon, \tau, X, Y, M} \operatorname{Trace}(M) \\
& {\left[\begin{array}{ccccccc}
\Theta_{1} & \tau I & \varepsilon D & \Theta_{2} & X Q & W^{T} R & X G^{T} \\
* & -\tau I & 0 & 0 & 0 & 0 & 0 \\
* & * & -\varepsilon I & 0 & 0 & 0 & 0 \\
* & * & * & -\varepsilon I & 0 & 0 & 0 \\
* & * & * & * & -Q & 0 & 0 \\
* & * & * & * & * & -R & 0 \\
* & * & * & * & * & * & -\tau I
\end{array}\right]<0,} \\
& {\left[\begin{array}{ll}
M & I \\
* & X
\end{array}\right]>0}
\end{aligned}
$$

has a solution $(\widetilde{\mathcal{\varepsilon}}, \widetilde{\tau}, \widetilde{X}, \widetilde{W}, \widetilde{M})$; then $u^{*}(t)=\widetilde{W} \widetilde{X}^{-1} x(t)$ will be the optimal state feedback GCC law for such system. More free variables were introduced into the problem above, so that the solution to (36) was less conservative.

\section{Nonfragile Guaranteed Cost Control Containing Nonlinear Perturbation}

In order to reduce the system tracking error, we suppose that the output $y_{d r}$ of system is constant vector which is a nonzero constant vector, and then the error vector is $e=y(t)-y_{d r}=$ $x(t)-y_{d r}$. So the error system can be deduced such that

$$
\begin{aligned}
\dot{e}= & \left(A_{1}+\Delta A\right) e+\left(B_{1}+\Delta B\right) u_{k}+\left(A_{1}+\Delta A\right) y_{d r} \\
& +f_{L}(x, t) .
\end{aligned}
$$


For formula (37), design controller is with guaranteed cost. Consider that reference state input $y_{d r}$ is bounded and assume that nonlinear function $f_{L}(e, \xi, t)=f_{L}(x(t))$ satisfies

$$
f_{L}^{T}(e, \xi, t) f_{L}(e, \xi, t) \leq e^{T}(t) G^{T} G e(t)
$$

where $G$ is a constant matrix. Meanwhile, it satisfies $G^{T} G>0$.

It is clear that the regulator of system (37) is equal to design of the tracking controller for system (2). Let $u(t)=$ $u_{k e}+v_{t r}=K e+v_{t r}$, and (37) can be expressed as

$$
\begin{aligned}
\dot{e}= & \left(\left(A_{1}+\Delta A\right)+\left(B_{1}+\Delta B\right) K\right) e+\left(B_{1}+\Delta B\right) v_{t r} \\
& +\left(A_{1}+\Delta A\right) y_{d r}+f_{L}(e, \xi, t) .
\end{aligned}
$$

To realize the regulation and control of system (37), it is requested that 0 is the balance point of this system; thus let

$$
\left(B_{1}+\Delta B\right) v_{t r}+\left(A_{1}+\Delta A\right) y_{d r}+f_{L}(e, \xi, t)=0 .
$$

If this system is progressively stable, then $u_{\infty}$ is approximated to meet formula (41).

When $t \rightarrow \infty, e(t) \rightarrow 0, \dot{e}(t) \rightarrow 0, f_{L}(e, \xi, t) \rightarrow 0$, $u(\infty) \rightarrow u_{\infty}=v_{t r}$, then get

$$
\left(B_{1}+\Delta B\right) u_{\infty}+\left(A_{1}+\Delta A\right) y_{d r}=0
$$

That is,

$$
v_{t r}=u_{\infty}=-B_{1}^{+} A_{1} y_{d r} .
$$

Let $K_{c}=-B_{1}{ }^{+} A_{1}$; a feedback controller with uncertainties is given as

$$
u(t)=v_{k}+v_{t r}=(K+\Delta K) e(t)+K_{c} y_{d r}
$$

where $K$ is state feedback matrix and $K_{c}$ is feedforward compensation matrix. $\Delta K$ is an uncertain matrix with corresponding dimension, which is norm-bounded in assumption and satisfies

$$
\Delta K=D_{K} N_{K}(t) E_{K},
$$

where $D_{K}, E_{K}$ are known matrices and $N_{K}(t)$ is an unknown time-varying matrix, represents a neural network control output, and satisfies

$$
N_{K}^{T}(t) N_{K}(t) \leq I
$$

Order $u_{e}(t)=u(t)-u_{\infty}$, and define quadratic performance index as the tracking performance of system; we have

$$
J_{e}=\int_{0}^{\infty}\left(e^{T}(t) Q e(t)+u_{e}^{T}(t) R u_{e}(t)\right) d t .
$$

Definition 10. For uncertain system (37) and cost function (46), $K^{*}$ can be defined as a nonfragile guaranteed cost control gain matrix with the corresponding upper bound $J^{*}$ of cost function, only if there exists a controller (43) satisfying inequality (45), which makes the closed-loop system with system uncertainties in (3) and nonlinear perturbation in (38) asymptotically stable, where $K^{*}$ is a constant gain matrix and $J^{*} \geq J$ is a positive constant.
Theorem 11. For an uncertain system (37) and cost function (46), if there exist symmetric positive definite matrices $P$ and $K$, with a scalar quantity $\varepsilon_{1}>0$, satisfying the following equation

$$
M=\left[\begin{array}{cc}
Q+\widetilde{K}^{T} R \widetilde{K}+\Lambda_{1}+\Lambda_{1}^{T}+\Lambda_{2} & P \\
P & -\varepsilon_{1}^{-1} I
\end{array}\right]<0,
$$

$\mathbf{K}$ is a nonfragile guaranteed cost control gain matrix and $J^{*}=$ $e_{0}^{T} P e_{0}$ is the upper bound of cost function (46), where

$$
\begin{aligned}
\Lambda_{1} & =P(\widetilde{A}+\widetilde{B} \widetilde{K}), \\
\Lambda_{2} & =\varepsilon_{1}^{-1} G^{T} G, \\
\widetilde{A} & =A+\Delta A, \\
\widetilde{B} & =B+\Delta B, \\
\widetilde{K} & =K+\Delta K .
\end{aligned}
$$

Proof. Select the Lyapunov function $V(t)=e^{T}(t) P e(t)$, where $P$ is a positive definite matrix, and based on the control law (43), the time derivative of $V(t)$ with respect to time $t$ yields [35]

$$
\dot{V}(e)=\left[\begin{array}{c}
e(t) \\
f_{L}(e, v, t)
\end{array}\right]^{T}\left[\begin{array}{cc}
\Lambda_{1}^{T}+\Lambda_{1} & P \\
P & 0
\end{array}\right]\left[\begin{array}{c}
e(t) \\
f_{L}(e, v, t)
\end{array}\right] .
$$

And (37) can be transformed into

$$
\left[\begin{array}{c}
e(t) \\
f_{L}(e, v, t)
\end{array}\right]^{T}\left[\begin{array}{cc}
-G^{T} G & 0 \\
0 & I
\end{array}\right]\left[\begin{array}{c}
e(t) \\
f_{L}(e, v, t)
\end{array}\right]<0 .
$$

According to matrix inequality (47), it can be concluded that

$$
\left[\begin{array}{c}
e(t) \\
f_{L}(e, v, t)
\end{array}\right]^{T} M\left[\begin{array}{c}
e(t) \\
f_{L}(e, v, t)
\end{array}\right]<0
$$

Multiply $\varepsilon_{1}$ and add (51) to the left side of (50); it can be derived as $\dot{V}(e)<-e^{T} Q e-e^{T} \widetilde{K}^{T} R \widetilde{K} e=-e^{T} Q e-v_{k}^{T} R v_{k}<$ 0 . According to Lyapunov stability theory, system (37) is asymptotically stable.

After the integration of (51) on both sides from $t=0$ to $t=$ $\infty$ and equation $e(\infty)=0$ inferred from asymptotic stability of the closed-loop system, it can be concluded that

$$
\begin{aligned}
J & =\int_{0}^{\infty} e^{T}\left(Q+\widetilde{K}^{T} R \widetilde{K}\right) e d t<V(e(0))-V(e(\infty)) \\
& =e_{0}{ }^{T} P e_{0}=J^{*}
\end{aligned}
$$

This completes the proof.

In order to carry out nonfragile guaranteed cost controller for the system, the equivalent LMI expression of condition (45) is given based on Theorem 12. 
Theorem 12. For given positive definite matrices $P$ and $K$, the closed-loop system (37) has a feasible solution $\left(\rho, \varepsilon_{1}, \varepsilon_{2}, Y, X\right)$, which guarantees the establishment of condition (47) for all allowable uncertainty. A nonfragile state feedback controller gain matrix $K=Y X^{-1}$ and an upper bound cost function $J \leq \operatorname{Trace}\left(X^{-1}\right)=\widehat{J}^{*}$ exist, if and only if there exist $\rho, \varepsilon_{1}$, $\varepsilon_{2}>0$, symmetric positive definite matrix $X$, and real matrix $Y$, such that the following LMI holds:

$$
\left[\begin{array}{cccccccc}
\Lambda+\rho D D^{T} & * & * & * & * & * & * & * \\
E_{1} X+E_{2} Y & -\rho I & * & * & * & * & * & * \\
X & 0 & -Q^{-1} & * & * & * & * & * \\
Y & 0 & 0 & -R^{-1} & * & * & * & * \\
E_{K} X & 0 & 0 & 0 & -\varepsilon_{2} I & * & * & * \\
\varepsilon_{2} D_{K}^{T} B^{T} & \varepsilon_{2} D_{K}^{T} E_{2}^{T} & 0 & \varepsilon_{2} D_{K}^{T} & 0 & -\varepsilon_{2} I & * & * \\
X & 0 & 0 & 0 & 0 & 0 & -\varepsilon_{1}\left(G^{T} G\right)^{-1} & * \\
\varepsilon_{1} I & 0 & 0 & 0 & 0 & 0 & 0 & -\varepsilon_{1} I
\end{array}\right]<0,
$$

where $\Lambda=A X+B Y+(A X+B Y)^{T}$ and $*$ is the corresponding symmetric part of the matrix.

Proof. Following proof process of Theorem 11, we can transform the existence condition (47) based on Lemmas 1 and 2 into the following expression:

$$
\left[\begin{array}{cccc}
\Lambda_{2}+\Lambda_{3}+\Lambda_{4}+\Lambda_{5} & * & * & * \\
E_{1}+E_{2} \widetilde{K} & -\rho I & * & * \\
I & 0 & -Q^{-1} & * \\
\widetilde{K} & 0 & 0 & -R^{-1}
\end{array}\right]<0,
$$

where $\Lambda_{3}=P(A+B \widetilde{K})+(A+B \widetilde{K})^{T} P, \Lambda_{4}=\rho P D D^{T} P$, $\Lambda_{5}=\varepsilon_{1} P^{T} P$. Substituting (44) into left side of (54), it can be decomposed as

$$
Y_{1}+\Sigma_{1}+\Sigma_{1}^{T}<0
$$

where

$$
\begin{aligned}
& Y_{1}=\left[\begin{array}{cccc}
\Lambda_{2}+\Lambda_{4}+G^{T} G & * & * & * \\
E_{1}+E_{2} K & -\rho I & * & * \\
I & 0 & -Q^{-1} & * \\
K & 0 & 0 & -R^{-1}
\end{array}\right] \text {, } \\
& \Sigma_{1}=\left[\begin{array}{c}
P B D_{K} \\
E_{2} D_{K} \\
0 \\
D_{K}
\end{array}\right] N_{K}\left[\begin{array}{llll}
E_{K} & 0 & 0 & 0
\end{array}\right] .
\end{aligned}
$$

According to matrix inequality lemma, for all matrices $N_{k}$ meeting $N_{K}^{T} N_{K} \leq I$ and a scalar $\varepsilon_{2}>0$, (55) is equivalent to

$$
\begin{aligned}
& Y_{1}+\varepsilon_{2}\left[\begin{array}{c}
P B D_{K} \\
E_{2} D_{K} \\
0 \\
D_{K}
\end{array}\right]\left[\begin{array}{c}
P B D_{K} \\
E_{2} D_{K} \\
0 \\
D_{K}
\end{array}\right]^{T}+\varepsilon_{2}^{-1}\left[\begin{array}{c}
E_{K}^{T} \\
0 \\
0 \\
0
\end{array}\right]\left[\begin{array}{c}
E_{K}^{T} \\
0 \\
0 \\
0
\end{array}\right]^{T} \\
& <0 \text {. }
\end{aligned}
$$

Applying Schur complement and multiplying each side of (57) by diag matrix $\left\{P^{-1}, I, I, I, I, \varepsilon_{2} I, I, \varepsilon_{1} I\right\}$, the linear matrix inequality (53) including variables $\rho, \varepsilon_{1}, \varepsilon_{2}$ and matrices $Y$ and $X$ can be obtained and parametric expression of the nonfragile guarantee cost control gain is given as $K=Y X^{-1}$, where $X=P^{-1}, Y=K P^{-1}$. Proof is then finished.

Theorem 13. For (37) and cost function (46), there exist an optimal nonfragile guaranteed cost control gain matrix $K^{*}=$ $Y^{*}\left(X^{*}\right)^{-1}$ and a minimal upper bound Trace $(S)$ of the cost function $J^{*}$, and the following optimization problem

$$
\min _{\rho, \varepsilon_{1}, \varepsilon_{2}, Y, X, S} \operatorname{Trace}(S)
$$

has a solution as $\left(\rho^{*}, \varepsilon_{1}{ }^{*}, \varepsilon_{2}{ }^{*}, Y^{*}, X^{*}, S^{*}\right)$, satisfying both the linear matrix inequality (53) and the following equation:

$$
\left[\begin{array}{ll}
S & * \\
I & X
\end{array}\right]>0
$$

Proof. If $\left(\rho^{*}, \varepsilon_{1}{ }^{*}, \varepsilon_{2}{ }^{*}, Y^{*}, X^{*}, S^{*}\right)$ is a feasible solution of (58), it is also feasible to (53). According to Theorems 11 and 12, $K^{*}=Y^{*}\left(X^{*}\right)^{-1}$ is a feasible nonfragile guaranteed cost control gain matrix for the system. And using Lemma 1, (59) is equivalent to $S>X^{-1}>0$, and the minimum of $\operatorname{Trace}(S)$ will ensure the minimization of $\operatorname{Trace}\left(X^{-1}\right)$, which is the minimization of cost function's upper bound. This completes the proof. 


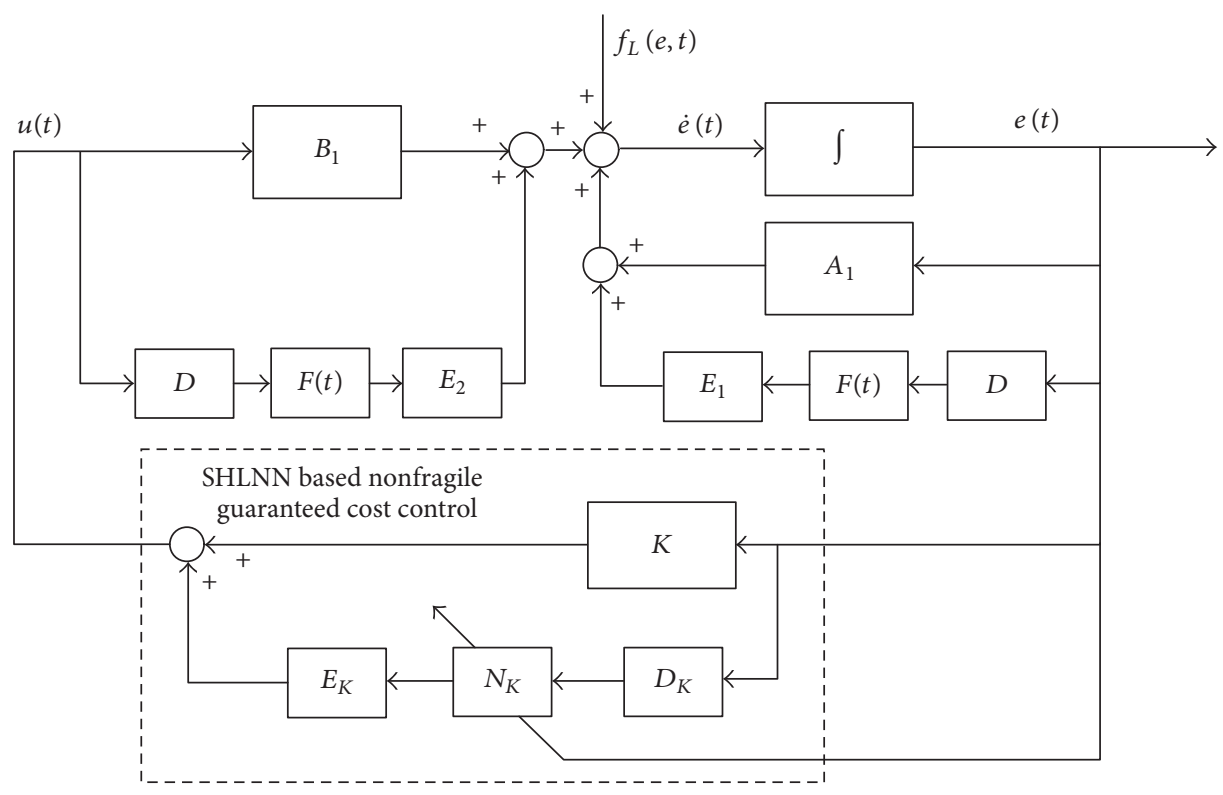

FIGURE 1: SHLNN based nonfragile robust control structure.

\section{SHLNN Controller Design for Nonfragile Guaranteed Cost Control Strategy}

SHLNN controller, with multilayer architecture, is composed of artificial neurons which simulate biologic ones. SHLNN can achieve mapping to arbitrary nonlinear function. The basic structure includes weighted summation, nonlinear function map, and linear dynamic states, and the inputoutput relationship is defined as follows $[36,37]$ :

$$
y(W, V, x)=g\left(W^{T} f\left(V^{T} x\right)\right) .
$$

Take the output of SHLNN $N_{K}(t)$ as additive gain perturbation of the gain matrix $K$ in (37); it will eliminate the influence of conservative nonfragile guaranteed cost control gains by online learning mechanism. As a result, the system error can quickly converge to zero and reduce the upper bound of cost function finally [38]. The stability of closedloop system is guaranteed by Theorem 12 . The cost function of NN controller is given as

$$
E(t)=\frac{1}{2} e^{T}(t) e(t) .
$$

If $E(t)$ can be minimized by SHLNN, the tracking error $e(t)$ will be reduced as small as possible correspondingly, and better tracking performance can be achieved in the system. As for (37), the proposed control structure is shown in Figure 1.

If $N_{K}(t)$ is used as network output, the constraint condition $N_{K}^{T}(t) N_{K}(t) \leq I$ should be satisfied, so modification of the SHLNN structure is needed. We choose hyperbolic tangent function $\sigma(\bullet)$ as activation function $g(\bullet)$ for output layer and keep the mapping relation $f(\bullet)$ unchanged in hidden layer, where

$$
f(z)=g(z)=\sigma(z)=\frac{e^{z}-e^{-z}}{e^{z}+e^{-z}} .
$$

Through the modification above, network outputs can be constrained between -1 and +1 by the hyperbolic tangent function $\sigma(\bullet)$, which satisfies $N_{K}^{T}(t) N_{K}(t) \leq I$.

Suppose $\Delta K$ can be transformed as

$$
\Delta K=\left[\begin{array}{c}
D_{K_{1}} \\
M \\
D_{K_{l}}
\end{array}\right]_{l \times m}^{T}\left[\begin{array}{ccc}
N_{K_{1}} & 0 & 0 \\
0 & O & 0 \\
0 & 0 & N_{K_{l}}
\end{array}\right]_{l \times l}\left[\begin{array}{c}
E_{K_{1}} \\
M \\
E_{K_{l}}
\end{array}\right]_{l \times n},
$$

where $N_{K}$ is a matrix of $l \times l$. Consider each SHLNN as an output; partial derivative of (43) can be expressed as

$$
\frac{\partial u(t)}{\partial N_{K_{i}}(t)}=D_{K_{i}} E_{K_{i}} e(t)=p(t), \quad(i=1, L, l) .
$$

If the system input is defined as vector $\pi$, we can describe neural network equations as $O_{V}=V^{T} \pi$ and $O_{W}=W^{T} \sigma\left(O_{V}\right)$, in which $\sigma_{W}$ and $\sigma_{V}$ represent derivatives of activation function to weighted matrices $W$ and $V$, respectively. According to the chain rule of SHLNN weight, an update scheme is inferred as follows:

$$
\begin{aligned}
& \dot{W}_{i}=\kappa_{W i} e \operatorname{sgn}\left(\frac{\partial e}{\partial u}\right) p \times \sigma \times \sigma_{W}^{T}+\lambda_{i} W_{i}, \\
& \dot{V}_{i}=\kappa_{V i} e \operatorname{sgn}\left(\frac{\partial e}{\partial u}\right) p \times \pi\left(\sigma_{V} \times W^{T} \sigma_{W}\right)^{T}+\lambda_{i} V_{i},
\end{aligned}
$$

where $\kappa_{W i}$ and $\kappa_{V i}$ stand for learning rates and $\lambda_{i}$ represents the inertial coefficient, $i=1, \ldots, l$. 


\section{Simulation Results Analysis}

7.1. State Regulating Simulation of Hypersonic Vehicle. For a specific hypersonic vehicle system, the relevant parameters of the system equation are given as follows.

$$
\begin{aligned}
& A_{1} \\
& =\left[\begin{array}{cccccc}
-0.0299 & 1.0000 & 0 & 0 & 0 & 0 \\
-0.6345 & -0.0184 & 0 & 0 & 0 & 0 \\
0 & 0 & -0.0058 & 0.9879 & 0 & \\
0 & 0 & -1.0467 & 0 & 0 & 0 \\
0 & 0 & 0 & 0 & 0 & 1.0000 \\
-0.0009 & 0 & 56.5463 & 0 & 0 & -0.0167
\end{array}\right],
\end{aligned}
$$$$
B_{1}=\left[\begin{array}{ccc}
-0.0008 & 0 & 0 \\
-4.3885 & 0 & 0 \\
0 & -0.0001 & 0.0001 \\
0 & -0.3703 & 1.9365 \\
0 & 0 & 0 \\
0 & 0.6356 & -27.4183
\end{array}\right] \text {, }
$$$$
D=\operatorname{diag}\left(\begin{array}{llllll}
1 & 1 & 1 & 1 & 1 & 1
\end{array}\right) \text {, }
$$

$E_{1}=\left[\begin{array}{cccccc}0 & 0 & 0 & 0 & 0 & 0 \\ 0.0346 & 0.0001 & 0.0001 & 0 & 0 & 0 \\ 0 & 0 & 0 & 0.0001 & 0 & 0.0009 \\ 0.0001 & 0 & 0.0088 & 0 & 0 & 0 \\ 0 & 0 & 0.01 & 0 & 0 & 0 \\ 0.0012 & 0 & 0.2974 & 0 & 0 & 0.0001\end{array}\right]$,

$E_{2}=\left[\begin{array}{ccc}0 & 0 & 0 \\ 0.0177 & 0 & 0 \\ 0 & 0 & 0 \\ 0 & 0.0015 & 0.0103 \\ 0 & 0 & 0 \\ 0 & 0.0025 & 0.1295\end{array}\right]$,

$Q=\operatorname{diag}\left(\begin{array}{llllll}20 & 0.1 & 20 & 0.1 & 20 & 0.1\end{array}\right)$,

$R=\operatorname{diag}\left(\begin{array}{lll}1 & 1 & 1\end{array}\right)$.

According to Theorem 6, matrix $K$ can be achieved.

\section{K}

$$
=\left[\begin{array}{cccccc}
7.3272 & 4.1847 & 0.0280 & 0.0079 & -0.0012 & 0.0007 \\
0.0091 & 0.0005 & 40.3491 & 19.6845 & -2.7003 & 1.0848 \\
0.0009 & 0.0002 & 4.3130 & 0.9314 & 3.2004 & 1.7965
\end{array}\right] .
$$

From the above equations, we can get the performance index $J^{*}=4.0560$ through Matlab simulation. Given the initial state vector $\mathbf{X}_{0}=\left[\begin{array}{llllll}0.2 & 0.1 & 0.1 & 0.1 & 0.1 & 0.1\end{array}\right]^{T}$, the simulation results of $\mathbf{x}(t)$ and $u(t)$ are shown in Figures 2 and 3, respectively. From Figure 2, six response curves of angles and angular velocities show that the regulating system is stable and controllable, with short settling time. Through the definition of $J(t)=\int_{0}^{t}\left[x^{T}(\tau) Q x(\tau)+u^{T}(\tau) R u(\tau)\right] d \tau$, the evolution process of $J(t)$ is shown in Figure 4, which shows that the system has a given performance index upper bound.

7.2. Robust Tracking Control Simulation Result of Hypersonic Vehicle. In numerical simulation, we decompose $\Delta A$ and $\Delta B$ like Section 7.1. Suppose there exists $G^{T} G=0.5 I$ in (38) and number of SHLNN network outputs in the matrix $N_{K}$ is three. Furthermore, in order to guarantee the ability to counteract system uncertainties, gain matrices $D_{K}$ and $E_{K}$ are given as

$$
\begin{aligned}
D_{K} & =\left[\begin{array}{ccc}
0.5 & 0 & 0 \\
0 & 0.5 & 0 \\
0 & 0 & 0.5
\end{array}\right], \\
E_{K} & =\left[\begin{array}{cccccc}
20 & 15 & 0 & 0 & 0 & 0 \\
0 & 0 & 10 & 5 & 0 & 0 \\
0 & 0 & 0 & 0 & 20 & 15
\end{array}\right] .
\end{aligned}
$$

So the gain matrix K can be obtained by LMI optimization approach according to Theorems 12 and 13.

$$
\begin{aligned}
& \mathbf{K} \\
& =\left[\begin{array}{ccccccc}
29.7482 & 22.4213 & 0.0166 & 0.0044 & -0.0012 & 0.0004 \\
0.0306 & 0.0004 & 74.1146 & 31.5391 & -8.0931 & -0.4972 \\
0.0008 & 0.0000 & 3.9106 & 0.7396 & 11.1610 & 8.4109
\end{array}\right] .
\end{aligned}
$$

Three SHLNN outputs are utilized to adjust gain coefficients of pitch, yaw, and roll, respectively. The network inputs are chosen as $\pi_{\alpha}=\left[\alpha_{c}, \dot{\alpha}_{c}, e_{\alpha}, u_{\alpha}\right]^{T}, \pi_{\beta}=\left[e_{\beta}, u_{\beta}\right]^{T}$, $\pi_{\gamma}=\left[\gamma_{c}, \dot{\gamma}_{c}, e_{\gamma}, u_{\gamma}\right]^{T}$, with predefined network outputs $N_{K i}$. Moreover, learning rates in (65) are $\kappa_{W 1}=\kappa_{V 1}=\kappa_{W 2}=$ $\kappa_{V 2}=0.4, \kappa_{W 3}=\kappa_{V 3}=0.2$, with inertial coefficients $\lambda_{1}=\lambda_{2}=\lambda_{3}=0.1$.

To test the tracking effect of the above control law, square waves are selected as the command of pitch and roll channels with the alterative amplitudes from $2.5^{\circ}$ to $5^{\circ}$ and $-10^{\circ}$ to $10^{\circ}$ correspondingly, with time period as 20 seconds. In Figure 5, tracking performance results of GCC method and the proposed scheme are presented, where dash lines stand for standard GCC method results and solid lines are the responses using nonfragile robust control strategy. Figure 6 denotes the three channel angular velocity curves and Figure 7 is the curves of elevator and rudder angles in pitch, yaw, and roll channels. Figure 8 illustrates the regulating process of the control gain matrix $K+\Delta K$ elements, where $\Delta K$ is adjusted by the three SHLNN outputs. As shown above, the tracking effect of nonfragile robust control gives a good improvement in dynamic performance and tracking errors are apparently decreased. Therefore, the nonfragile guaranteed cost control method, integrated with SHLNN controller to update gain values, is effective in improving the control performance as proposed. 


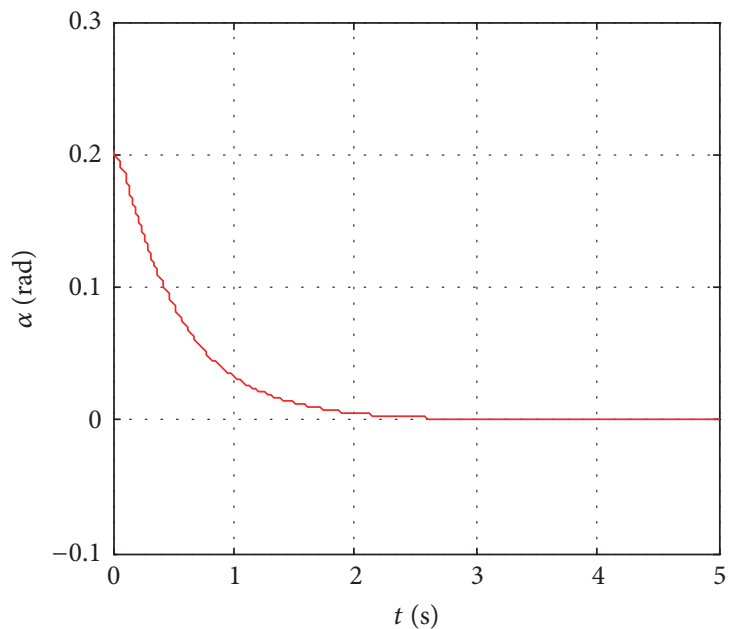

(a) Angle of attack curve

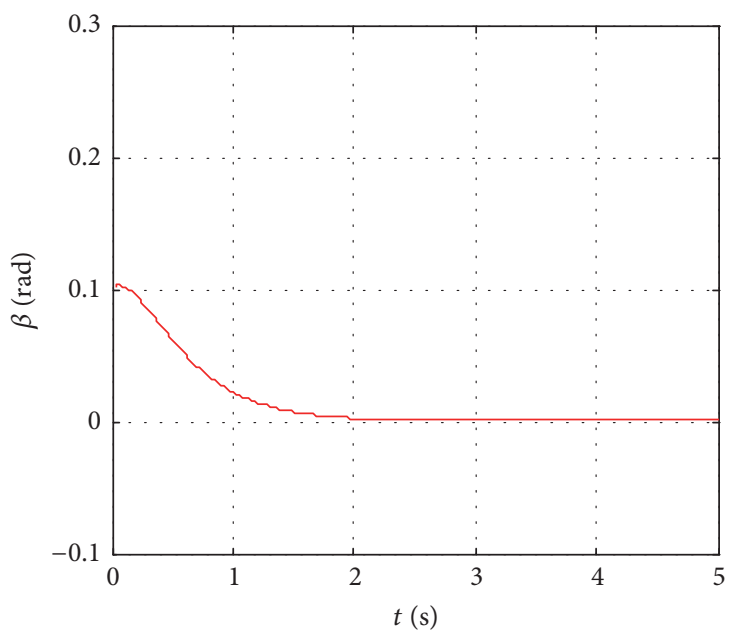

(c) Sideslip angle curve

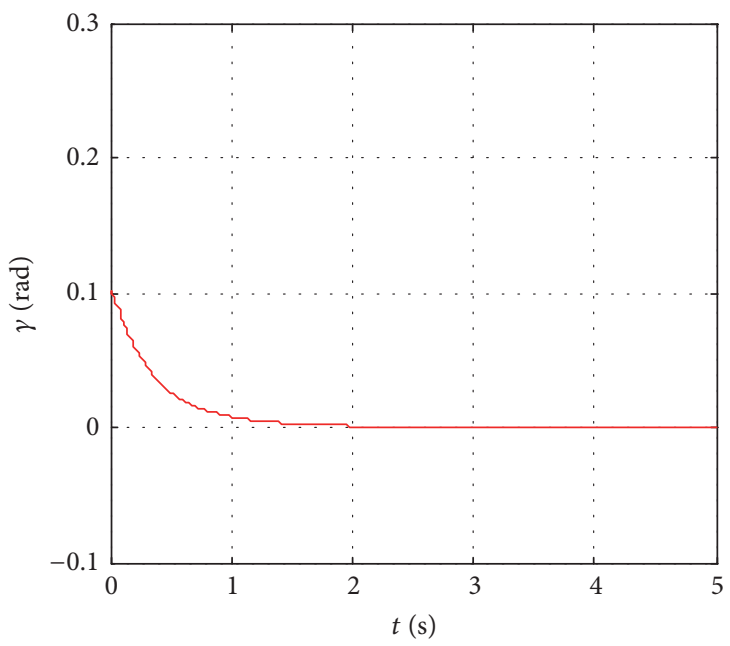

(e) Roll angle curve

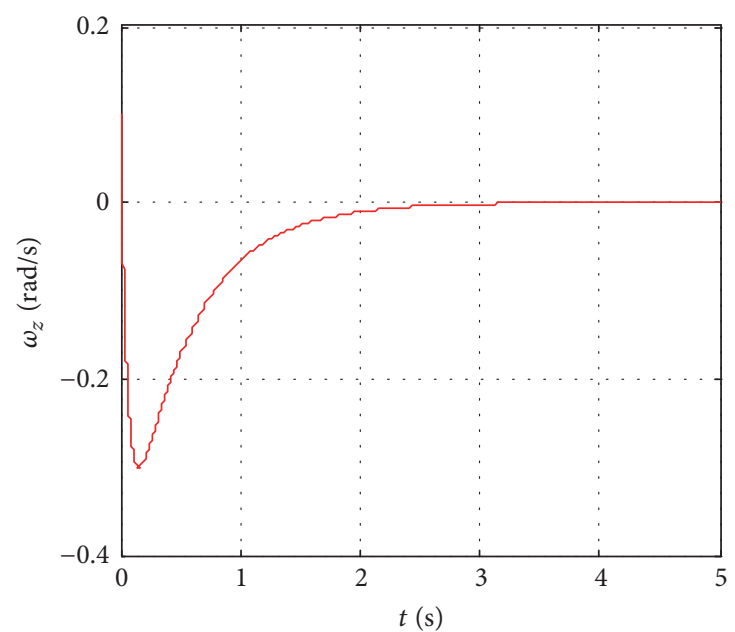

(b) Pitch angle velocity curve

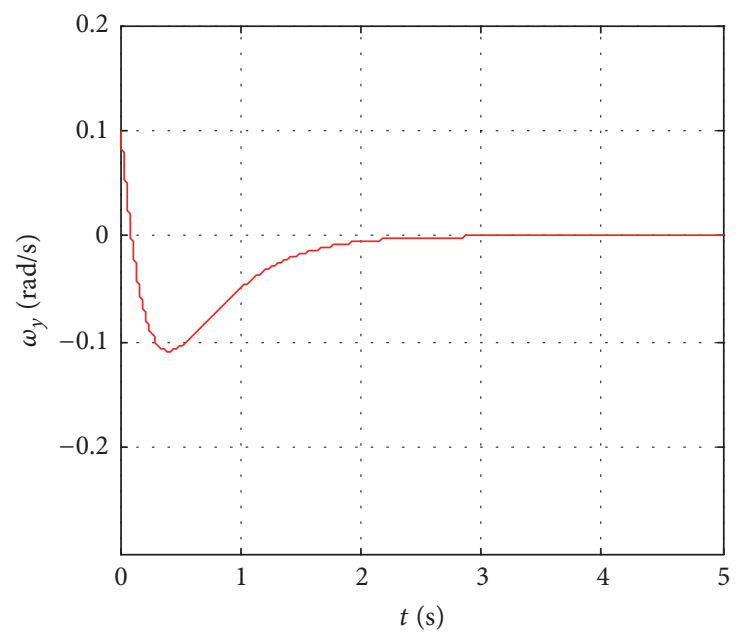

(d) Yaw angular velocity curve

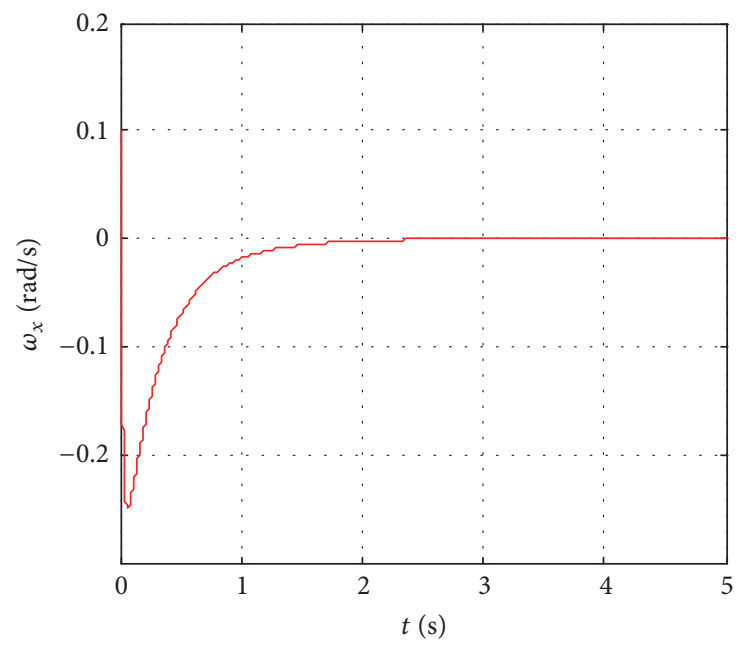

(f) Roll angular velocity curve

FIGURE 2: Responses of GCC controller. 


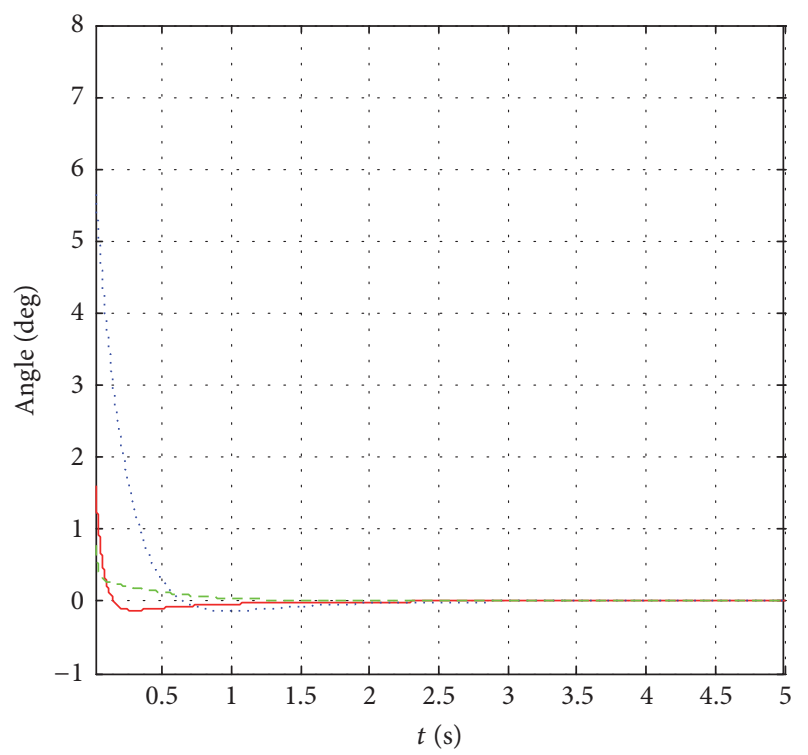

$-\delta_{\varphi}$
$\ldots \ldots \delta_{\psi}$
$---\delta_{\gamma}$

FIGURE 3: Regulating process of rudder angles as control inputs.

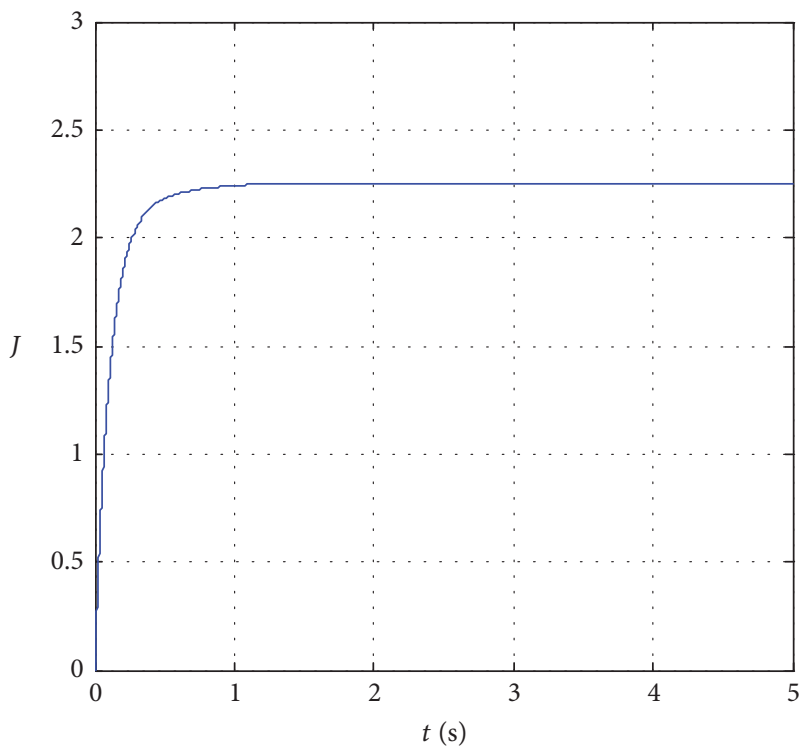

FIgURE 4: Performance index regulating process.

\section{Conclusions}

On the basis of RGCC design theory, the state feedback control law is obtained in this paper by applying Lyapunov function and satisfying overall Lipschitz condition. Specifically, the state-related nonlinear equation is built with a nonlinear part. The equations establishment formulates the process for solving the solution with LMI. Furthermore, adaptive SHLNN based nonfragile guaranteed cost control strategy is utilized to design the robust controller, with equivalent solution derived from LMI optimization approach,

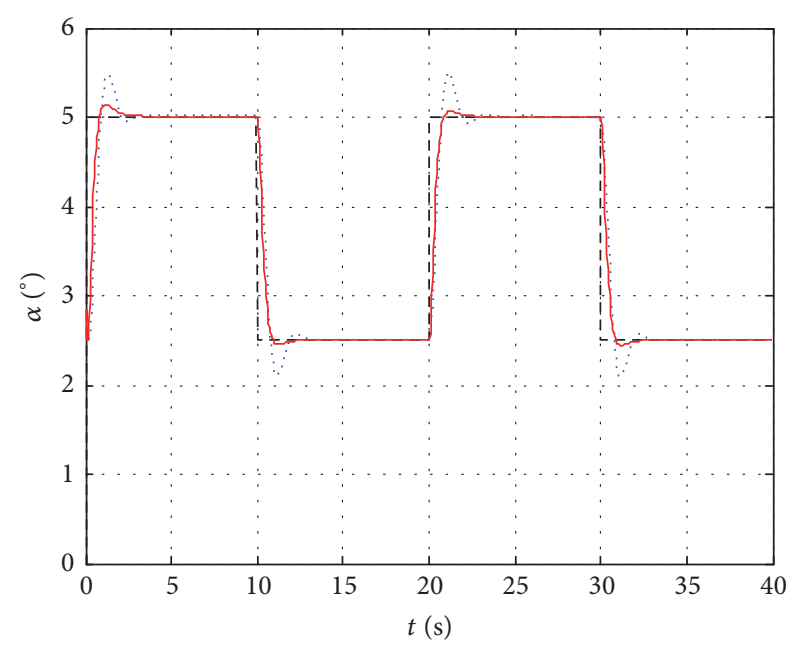

(a) Tracking response of attack angle

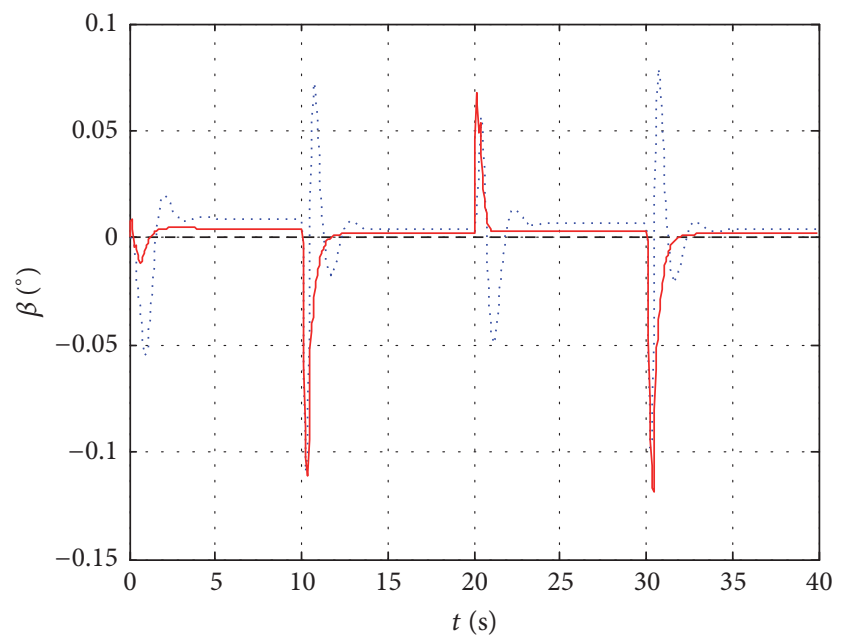

(b) Regulation response of sideslip angle

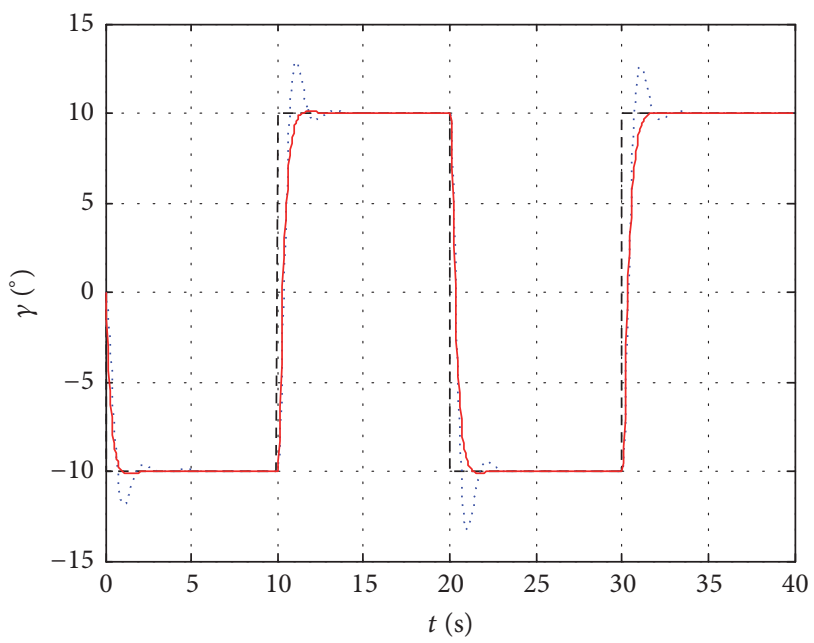

(c) Tracking response of roll angle

FIGURE 5: Tracking curves of three channel angles.

where SHLNN are exploited as additive gain adjustments to eliminate the influence of conservative control gains and 


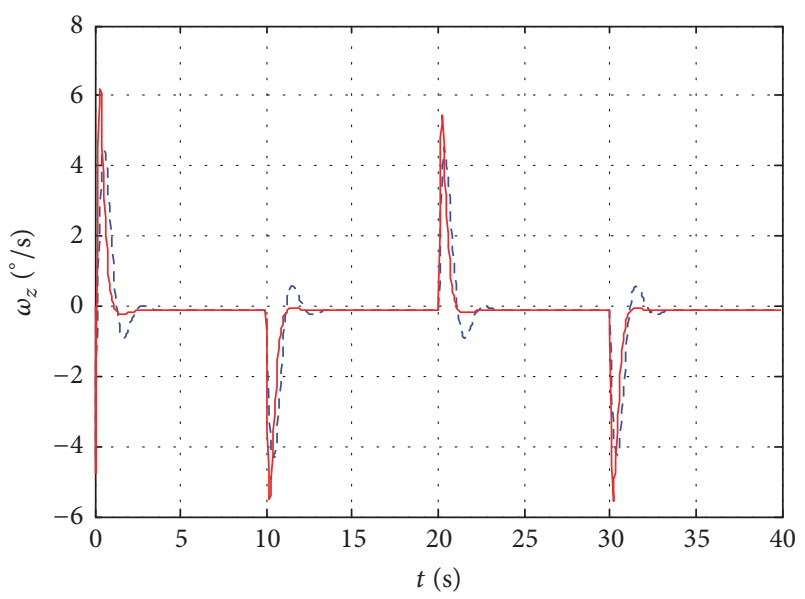

(a) Regulation response of pitch angular velocity

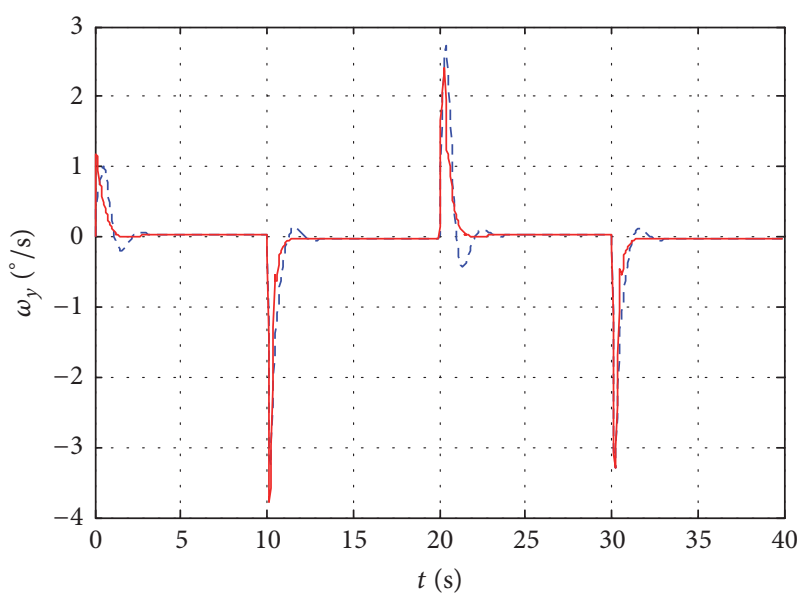

(b) Regulation response of yaw angular velocity

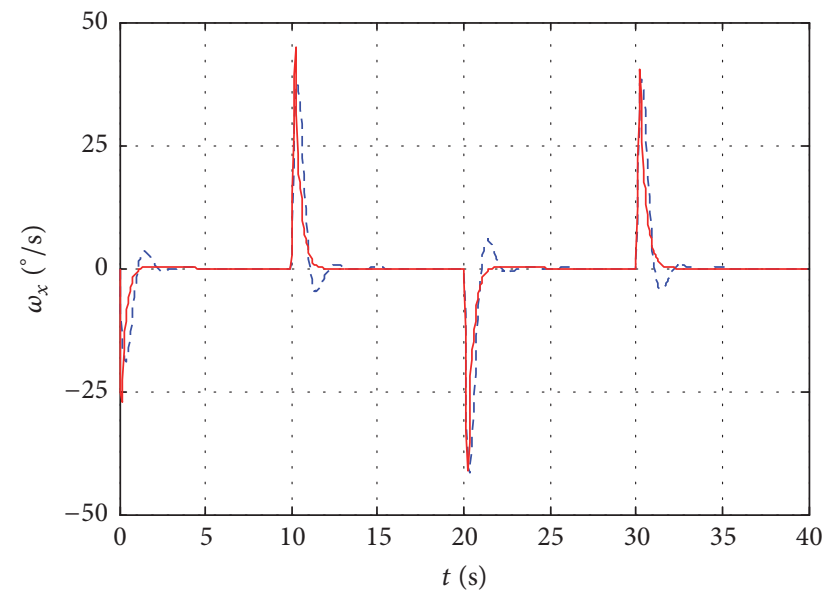

(c) Regulation response of roll angular velocity

FIGURE 6: Regulation curves of angular velocities.

counteract excessive upper bound of cost function caused by uncertainties. Finally, simulation verifications are carried out with a specific model of hypersonic vehicle, and feasibility

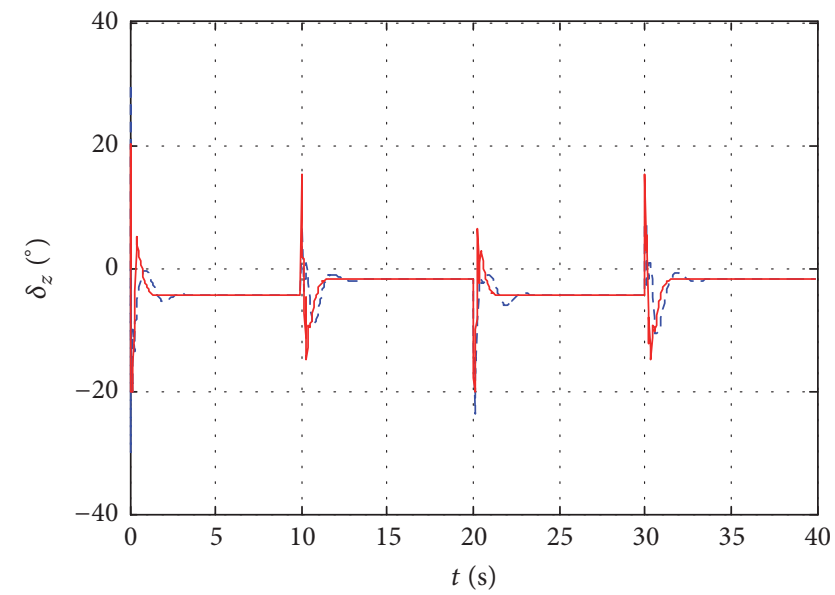

(a) Regulation curve of elevator angle in pitch channel

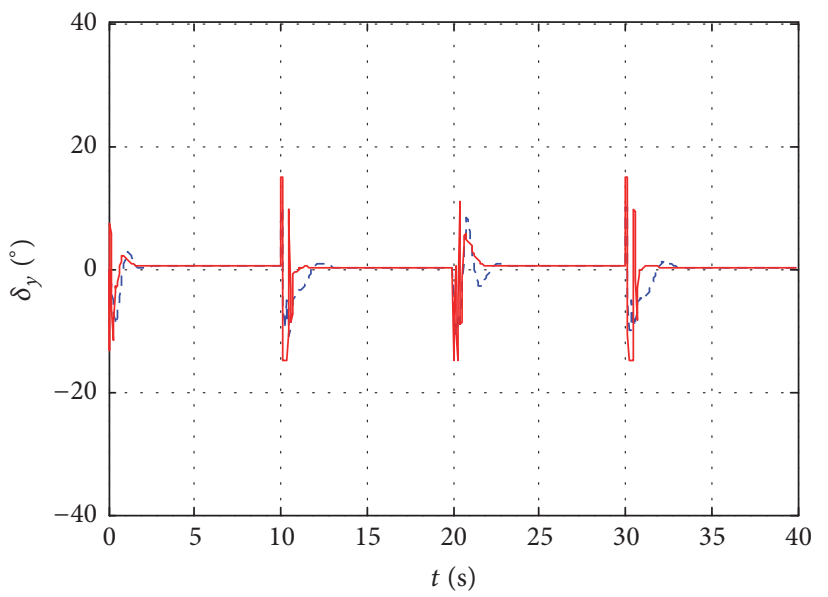

(b) Regulation curve of rudder angle in yaw channel

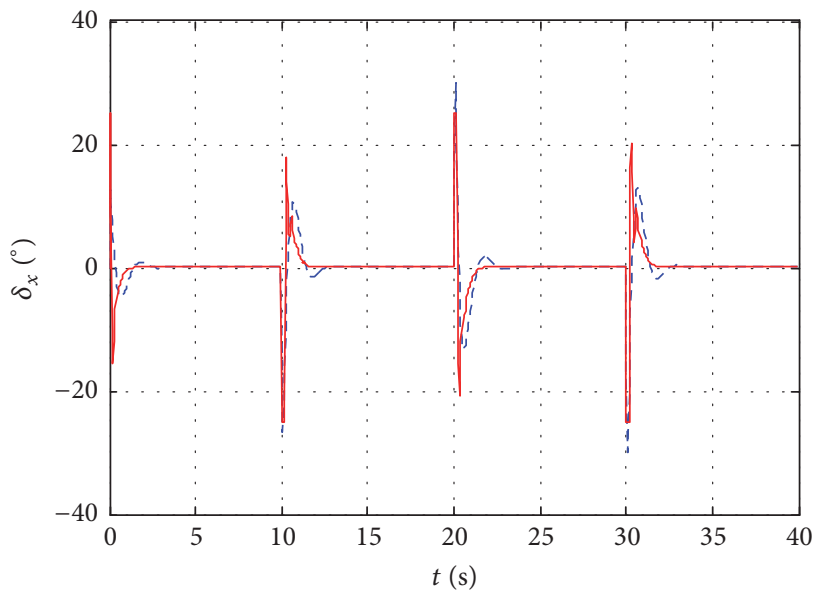

(c) Regulation curve of elevator angle in roll channel

FIGURE 7: Regulation curves of elevator and rudder angles in three channels.

and adaptability of the proposed algorithm are demonstrated accordingly, where the proposed method has better tracking performance in attitude control on the vehicle. 


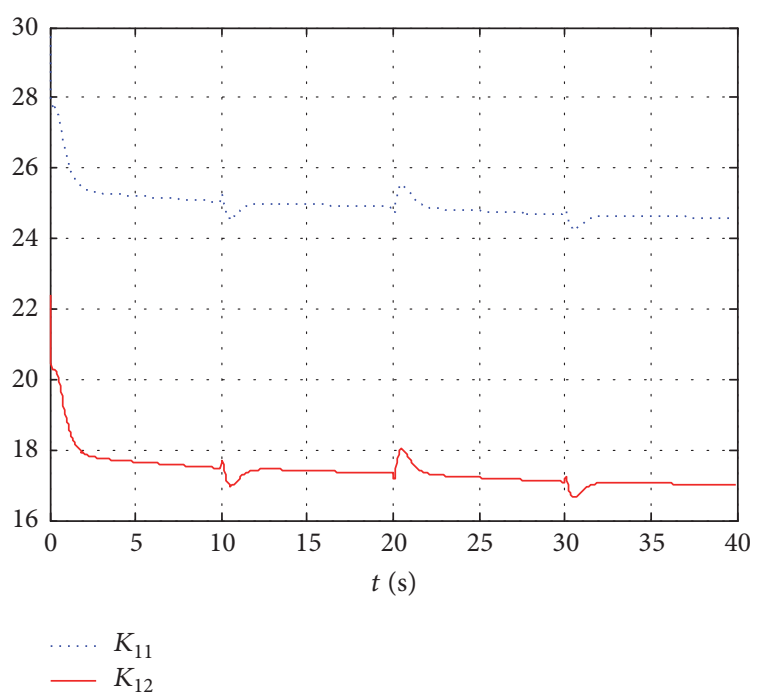

(a) Gains variation in pitch channel
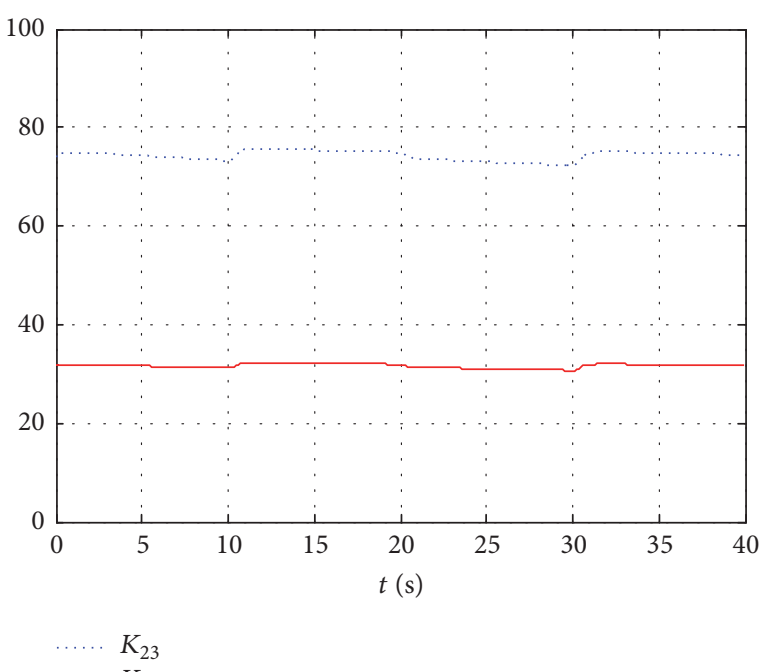

(b) Gains variation in yaw channel

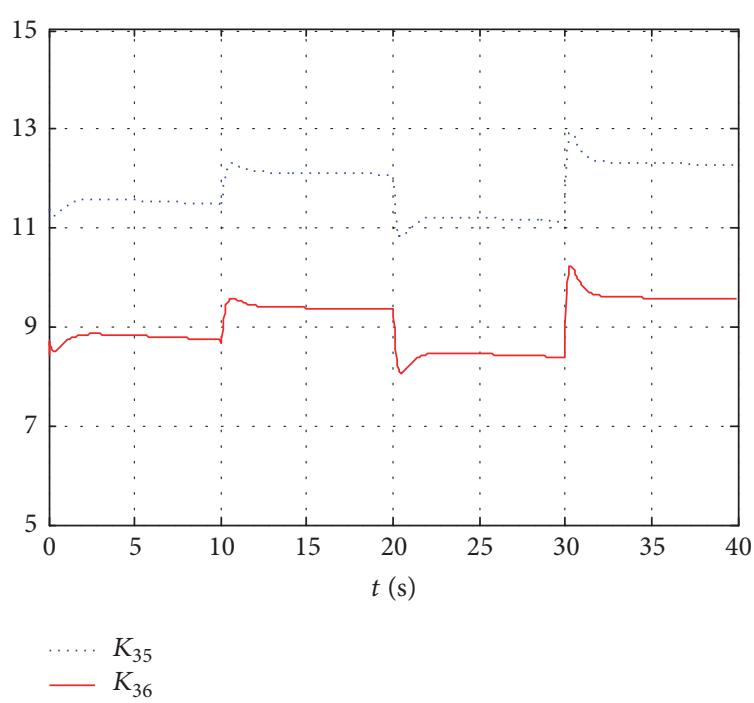

(c) Gains variation in roll channel

FIgURE 8: Control gains variation in three channels.

\section{Conflicts of Interest}

The authors declare that there are no conflicts of interest regarding the publication of this paper.

\section{Acknowledgments}

This work was supported in part by the National Natural Science Foundation of China $(61473124,61503173)$, the Science and Technology Research Project of Education Department of Henan Province (15A413016), and the Science and Technology Key Project of Henan Province (162102410051). This paper was also supported by the National Scholarship Fund.

\section{References}

[1] R. A. Hess, "Frequency domain-based pseudosliding mode flight control design," Journal of Aircraft, vol. 49, no. 6, pp. 20772088, 2012.

[2] H. Sun, S. Li, and C. Sun, "Finite time integral sliding mode control of hypersonic vehicles," Nonlinear Dynamics, vol. 73, no. 1-2, pp. 229-244, 2013.

[3] D. Liu, C. Wu, Q. Zhou, and H.-K. Lam, "Fuzzy guaranteed cost output tracking control for fuzzy discrete-time systems with different premise variables," Complexity, vol. 21, no. 5, pp. 265276, 2016.

[4] Z. Jin, J. Chen, Y. Sheng, and X. Liu, "Neural network based adaptive fuzzy PID-type sliding mode attitude control for a reentry vehicle," International Journal of Control, Automation, and Systems, vol. 15, no. 1, pp. 404-415, 2017.

[5] Y. Hou and S. Tong, "Adaptive fuzzy backstepping control for a class of MIMO switched nonlinear systems with unknown control directions," Complexity, vol. 21, no. 6, pp. 155-166, 2016.

[6] J. Na, G. Herrmann, and K. Zhang, "Improving transient performance of adaptive control via a modified reference model and novel adaption," International Journal of Robust and Nonlinear Control, vol. 27, no. 8, pp. 1351-1372, 2017.

[7] D. D. Donalson and C. T. Leondes, "A model referenced parameter tracking technique for adaptive control systems: $\mathrm{i}$ - the principle of adaptation," IEEE Transactions on Applications and Industry, vol. 82, no. 68, pp. 241-252, 1963.

[8] S. S. Dunn and R. Edelmann, "Minimum power spacecraft attitude control laws for small constant disturbance torques," IEEE Transactions on Automatic Control, vol. 13, no. 6, pp. 691694, 1968.

[9] G. Kreisselmeier and B. D. Anderson, "Robust model reference adaptive control," Institute of Electrical and Electronics Engineers Transactions on Automatic Control, vol. 31, no. 2, pp. 127-133, 1986.

[10] L. Giovanini, "Robust adaptive control using multiple models, switching and tuning," IET Control Theory and Applications, vol. 5, no. 18, pp. 2168-2178, 2011.

[11] Z. Pu, R. Yuan, X. Tan, and J. Yi, "Active robust control of uncertainty and flexibility suppression for air-breathing hypersonic vehicles," Aerospace Science and Technology, vol. 42, pp. 429441, 2015.

[12] J. Na, X. Ren, and D. Zheng, "Adaptive control for nonlinear pure-feedback systems with high-order sliding mode observer," IEEE Transactions on Neural Networks and Learning Systems, vol. 24, no. 3, pp. 370-382, 2013. 
[13] C. Peng, J. Zhang, and Y. C. Yan, "Adaptive event-triggering $H_{\infty}$ inf load frequency control for network-based power systems," IEEE Transactions on Industrial Electronics, no. 99, 2017.

[14] R. Matušů and R. Prokop, "Computation of robustly stabilizing PID controllers for interval systems," SpringerPlus, vol. 5, no. 1, article no. 702, 2016.

[15] E. Gershon, "Robust Reduced-order H-infinity Output-Feedback Control of Retarded Stochastic Linear Systems," IEEE Transactions on Automatic Control, vol. 58, no. 11, pp. 28982904, 2013.

[16] L. Liu, S. Dong, Y. Wang, and L. Ou, "Clearance of flight control law based on structural singular value theory," IEEE Transactions on Aerospace and Electronic Systems, vol. 51, no. 3, pp. 21382147, 2015.

[17] C. F. Li, Z. X. Ye, Y. J. Wang, and L. Liu, "Design of attitude decoupling control system for BTT missile using quantitative feedback theory," in Proceedings of the 2010 International Conference on Modelling, 2010.

[18] L. Denis-Vidal, C. Jauberthie, and G. Joly-Blanchard, "Identifiability of a nonlinear delayed-differential aerospace model," Institute of Electrical and Electronics Engineers Transactions on Automatic Control, vol. 51, no. 1, pp. 154-158, 2006.

[19] Y. Yuan, Y. Hu, and F. Sun, "Mixed H2/Ho control using a fuzzy singularly perturbed model with multiple perturbation parameters for gust load alleviation," Tsinghua Science and Technology, vol. 16, no. 4, pp. 344-351, 2011.

[20] S. Das and K. Halder, "Missile attitude control via a hybrid LQG-LTR-LQI control scheme with optimum weight selection," in Proceedings of the 1st International Conference on Automation, Control, Energy and Systems, ACES '14, pp. 1-6, February 2014.

[21] S. S. L. Chang and T. K. C. Peng, "Adaptive guaranteed cost control of systems with uncertain parameters," IEEE Transactions on Automatic Control, vol. 17, no. 4, pp. 474-483, 1972.

[22] H. Li, Y. Si, L. Wu, X. Hu, and H. Gao, "Guaranteed cost control with poles assignment for a flexible air-breathing hypersonic vehicle," International Journal of Systems Science, vol. 42, no. 5, pp. 863-876, 2011.

[23] Z. Deng, Y. Wang, L. Liu, and Q. Zhu, "Guaranteed cost decoupling control of bank-to-turn vehicle," IET Control Theory \& Applications, vol. 4, no. 9, pp. 1594-1604, 2010.

[24] E. Gyurkovics, "Guaranteed cost control of discrete-time uncertain systems with both state and input delays," International Journal of Control, vol. 89, no. 10, pp. 2073-2082, 2016.

[25] Z. Xu, X. Nian, H. Wang, and Y. Chen, "Robust guaranteed cost tracking control of quadrotor UAV with uncertainties," ISA Transactions $^{\circledR}$, vol. 69, pp. 157-165, 2017.

[26] L. Hu, F. Chen, B. Jiang, and G. Tao, "Control strategy for a quadrotor helicopter with state delay via improved guaranteed cost control and quantum adaptive control," Journal of Aerospace Engineering, vol. 30, no. 4, 2017.

[27] H.-N. Wu, M.-M. Li, and L. Guo, "Finite-horizon approximate optimal guaranteed cost control of uncertain nonlinear systems with application to Mars entry guidance," IEEE Transactions on Neural Networks and Learning Systems, vol. 26, no. 7, pp. 14561467, 2015.

[28] N. Sakthivel, R. Rakkiyappan, and J. H. Park, "Non-fragile synchronization control for complex networks with additive timevarying delays," Complexity, vol. 21, no. 1, pp. 296-321, 2015.

[29] A. Tandon and A. Dhawan, "Non-fragile robust optimal guaranteed cost control of uncertain 2-D discrete state-delayed systems," International Journal of Systems Science, vol. 47, no. 14, pp. 3303-3319, 2016.
[30] Y. Kong, D. Zhao, B. Yang, C. Han, and K. Han, "Robust non-fragile $\mathrm{H}$-infinity/L-2-L-infinity control of uncertain linear system with time-delay and application to vehicle active suspension," International Journal of Robust and Nonlinear Control, vol. 25, no. 13, pp. 2122-2141, 2015.

[31] S. X. Guo, "Robust reliability method for non-fragile guaranteed cost control of parametric uncertain systems," Systems and Control Letters, vol. 64, pp. 27-35, 2014.

[32] P. Sun and S. Wang, "Redundant input guaranteed cost nonfragile tracking control for omnidirectional rehabilitative training walker," International Journal of Control, Automation, and Systems, vol. 13, no. 2, pp. 454-462, 2015.

[33] L. Yu, Robust Control-Linear Matrix Inequality Method, Tsinghua University Press, Beijing, China, 2002.

[34] C. F. Li, L. Liu, Y. J. Wang, and Z. S. Wang, "Design of robust h-infinity control law via lmi for lifting vehicle," Information-an International Interdisciplinary Journal, vol. 15, no. 1, pp. 149-156, 2012.

[35] C. Peng and J. Zhang, "Delay-distribution-dependent load frequency control of power systems with probabilistic interval delays," IEEE Transactions on Power Systems, vol. 31, no. 4, pp. 3309-3317, 2016.

[36] J. Na, Q. Chen, X. Ren, and Y. Guo, "Adaptive prescribed performance motion control of servo mechanisms with friction compensation," IEEE Transactions on Industrial Electronics, vol. 61, no. 1, pp. 486-494, 2014.

[37] C. Yang, Y. Jiang, Z. Li, W. He, and C.-Y. Su, "Neural control of bimanual robots with guaranteed global stability and motion precision," IEEE Transactions on Industrial Informatics, 2017.

[38] G. Li, "Nonlinear model predictive control of a wave energy converter based on differential flatness parameterisation," International Journal of Control, vol. 90, no. 1, pp. 68-77, 2017. 


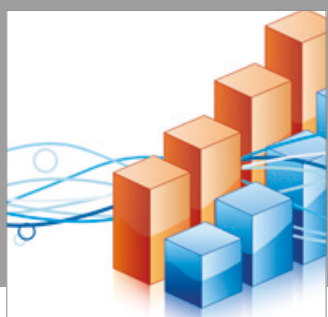

Advances in

Operations Research

vatersals

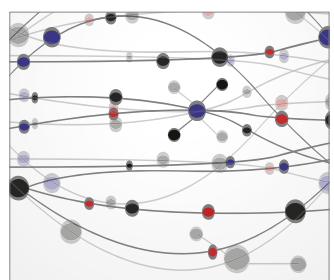

\section{The Scientific} World Journal
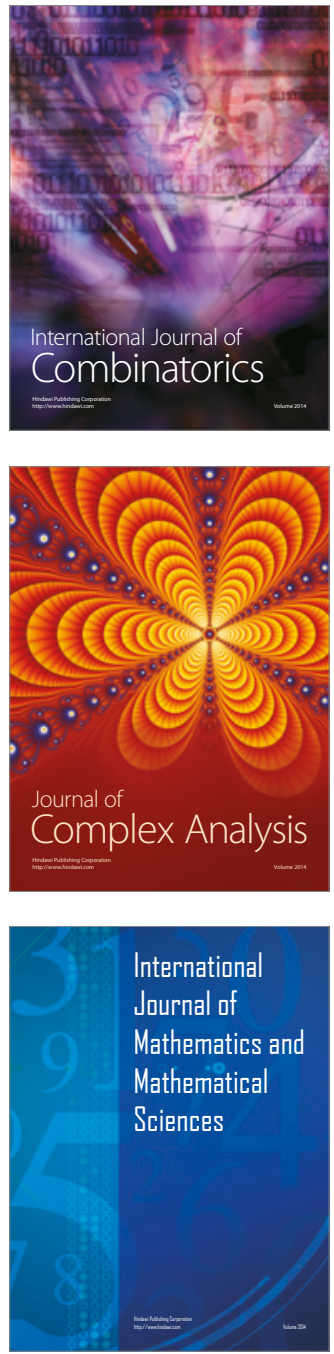
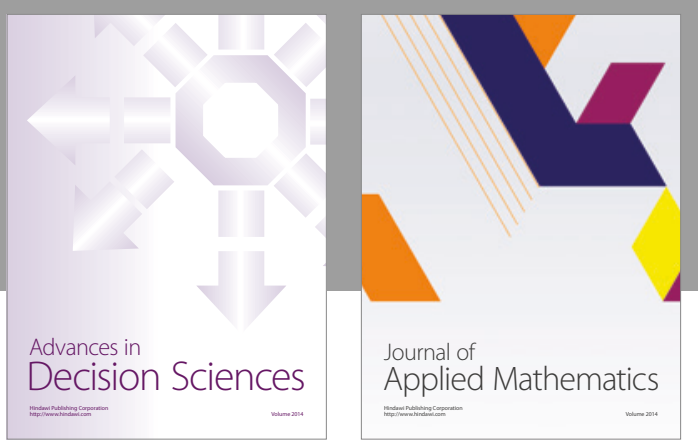

Algebra

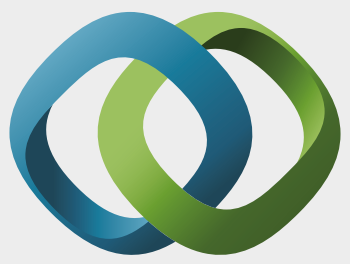

\section{Hindawi}

Submit your manuscripts at

https://www.hindawi.com
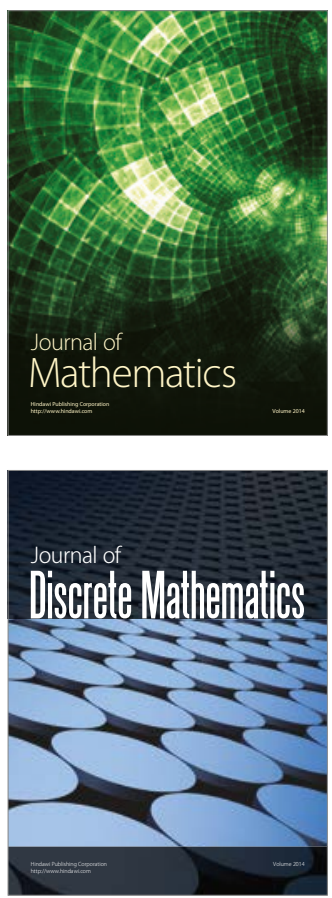

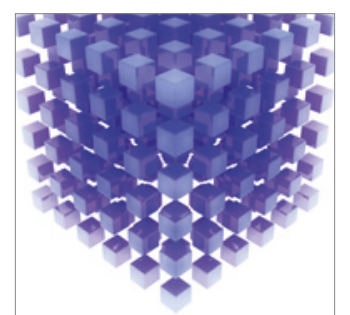

Mathematical Problems in Engineering
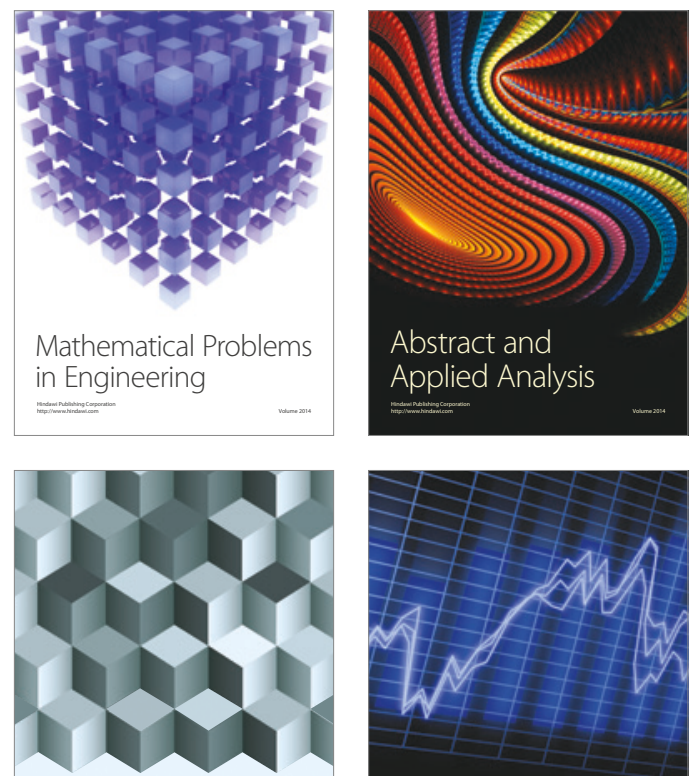

Journal of

Function Spaces

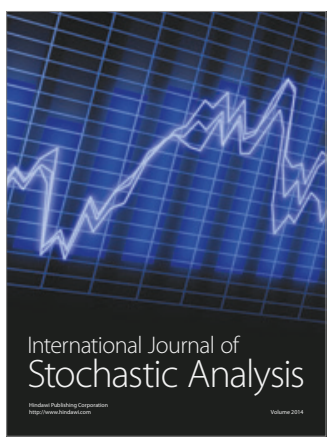

Probability and Statistics
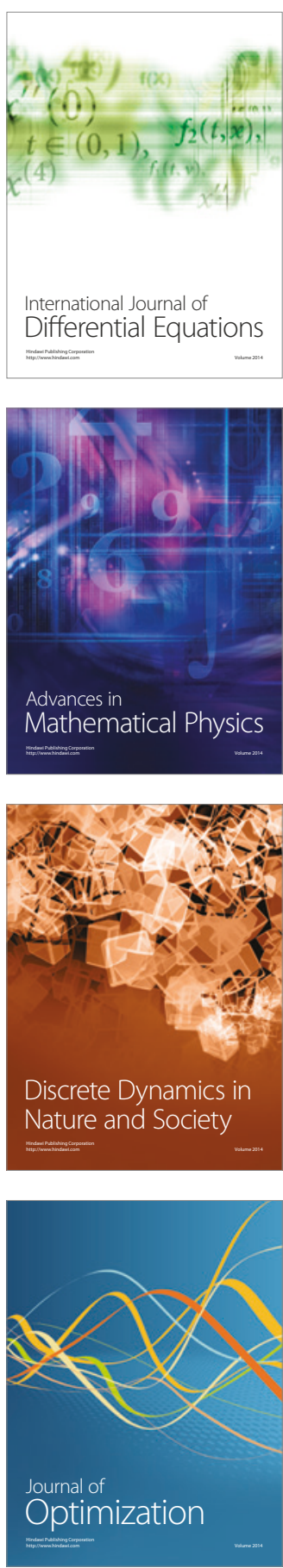